\title{
Correlations between Damage Indices and Seismic Parameters for Near-Field Earthquake Records
}

\author{
Soleymani, A. ${ }^{*}$ and Safi, M. ${ }^{1}$
}

\begin{abstract}
Damage assessments of concrete structures are essential to determine their seismic performances under earthquake events. In this paper, six three bays two dimensional concrete frames, five to ten story high, were analyzed under 30 near-field earthquake records by nonlinear dynamic analyses. The seismic performances were evaluated using two important overall damage indices which are: the modified Park-Ang index and the maximum softening damage index. The correlations between several seismic parameters and the two damage indices of the six concrete frames were determined using Spearman correlation coefficient. In the end, the values of correlations between seismic parameters and these two damage indices were compared and discussed. Subsequently, seismic parameters that have the strongest and the fairest correlation were presented. The numerical results of correlations have shown that Velocity Spectrum Intensity (VSI) has a strong interdependency with two overall structural damage indices (the modified Park-Ang model and the maximum softening damage) for all of the reinforced concrete frames.
\end{abstract}

Keywords: Correlation coefficient; damage indices; seismic parameters; seismic performance.

\section{Introduction}

The quantities of damages induced by the earthquake are important criteria to determine seismic performance of structures. For this purpose, damage indices can be used as a useful indicator to determine quantities of damages. Damage index may be calculated for single member of structure (local damage index) or the structure as a whole (global damage index). Two important global structural damage indices are the maximum softening damage index [1] and the overall modified Park-Ang damage index [2]. In this paper, the quantities of damages are calculated by these two damage indices.

There are important parameters to characterize the ground motion damaging potential. These seismic parameters may be of a simple instrumental peak value or a complicated mathematical derivation. In this paper, important seismic parameters including Peak Ground Acceleration (PGA), Peak Ground Velocity (PGV), Peak Ground Displacement (PGD),

\footnotetext{
${ }^{1}$ Department of Civil Engineering, Shahid Abbaspour Campus of Shahid Beheshti University, Tehran, IRAN

*Correspponding author, e-mail: abed.soleymani@yahoo.com; mohsafi@yahoo.com; Tel: +9809121023440
}

Note: Discussion is expected before November, $1^{\text {st }} 2014$, and will be published in the "Civil Engineering Dimension" volume 17, number 1, March 2015.

Received 28 August 2013; revised 20 March 2014; accepted 15 May 2014.
Velocity Spectrum Intensity (VSI), Housner Intensity (IH), Sustained Maximum Acceleration (SMA), Sustained Maximum Velocity (SMV), Effective Design Acceleration (EDA), Root-Mean-Square of Acceleration (ARMS), Root-mean-square of Velocity (VRMS), Root-mean-square of Displacement (DRMS), Arias Intensity (IA), Characteristic Intensity (IC), Specific Energy Density (SED), and Cumulative Absolute Velocity (CAV) have been used. Characterizations of seismic parameters that are mentioned above are presented in literatures [3-8].

Interdependencies between seismic parameters and damage indices can be used as a good indicator to predict the damaging potential of earthquake records. Elenas [9] has shown that the Pearson and the Spearman correlation coefficient have the same interdependency grade between some seismic parameters and two overall structural damage indices (the modified Park-Ang model [2] and the maximum softening DiPasquale and Cakmak model [1]).

Selected seismic parameters included peak parameters (e.g. PGV, PGD), spectral parameters (e.g. response, energy, Fourier-spectra) and energy parameters (e.g. ARIAS intensity, HUSID diagrams, Strong Motion Duration (SMD) after Trifunac and Brady [10], power P0.90). Among the seismic parameters, the spectral pseudo-acceleration and the spectral absolute seismic input energy have the strongest interdependencies with these two models. On the other hand, the PGA, the central period and the strong motion duration defined after Trifunac 
and Brady [10] exhibit poor interdependencies with these two models, only one eight story concrete frame was utilized instead of a range of different concrete frames [9].

Elenas and Meskouris [11] have shown that the peak ground motion parameters provide poor or fair correlation with the modified global Park-Ang model, the maximum floor acceleration, and the maximum inter-story drift. On the other hand, the spectral and energy parameters provide good interdependencies with these three damage indices. It was reported that period and the strong motion duration after Trifunac and Brady show poor interdependencies with these damage indices [11]. Also, only one eight story concrete frame similar to research done by Elenas [9] was utilized.

Nanos et al. [12] have evaluated interdependencies between several strong motion duration definitions and the overall building damage indices including Park-Ang model and DiPasquale and Cakmak model.

It has been shown that strong motion duration definitions that are not directly enclosing an accelerogram intensity measure, are inappropriate seismic damage potential descriptors [12]. Elenas [13] has investigated the interdependencies between the seismic intensity parameters and the structural damage indices. The modified Park-Ang damage index and drift model as damage index were used. It was concluded that the spectral and energy parameters provide strong correlation to the damage indices [13].

Safi and Soleymani [14] have estimated interdependencies between three global damage indices (the Bracci index, the modified flexural damage index and the drift index) and seismic parameters, time-variations of the members' degradations were also presented. It has been shown that the Housner intensity has the best correlations with these three damage indices [14].

In most papers, evaluations of correlations between damage indices and seismic parameters are focused on one reinforced concrete frames and a limited number of seismic records. One part of this paper consists of calculating two overall damage indices (the modified Park-Ang index and the maximum softening damage index) for 30 near-field earthquake records which are listed in Table 1. Other part of this paper includes determination of interdependencies between all of the calculated damage indices and important seismic parameters stated above. To increase the accuracy of the results, assessments were done for six different reinforced concrete frames (Figure 1).

\section{Damage Indices}

Damage indices are widely used to estimate the quantities of damages during and after the earthquake. In fact, the expression of damage in quantitative form is essential to estimate the maximum damage which is sustained by structure during an earthquake. Estekanchi and Arjomandi [15] investigated correlation between numerical values of damage indices which are based on deformation, energy, modal parameters, and low cycle fatigue behavior.

The maximum softening damage index is presented by DiPasquale and Cakmak [1]. This model calculates softening index relating the initial fundamental period of the structure to the final one. In fact, the concept of the maximum softening damage index is based on the variation of the vibrational periods during earthquake event. It is presented by the following expression:

$D I_{m}=1-\frac{T_{0}}{T_{\max }}$

Where, $D I_{m}$ is the maximum softening damage index, $T_{0}$ is the initial natural period of the structure and $T_{\max }$ is the maximum natural period of an equivalent linear system.

One of the important damage indices that have an extensive use is the Park-Ang model [16]. Because the Park-Ang damage index includes the effect of hysteretic energy dissipation and the effect of damage caused by excessive deformation, it is conceptually important and attractive. When inelastic behavior is restricted to plastic zone near the end of members, the relationship between local plastic rotation and member deformation can be presented by the modified Park-Ang model [2]. It is defined by the following formula:

$D I_{p}=\frac{\theta_{m}-\theta_{r}}{\theta_{u}-\theta_{r}}+\frac{\beta}{M_{y} \theta_{u}} E_{h}$

Where, $\theta_{m}$ is maximum rotation related to loading history; $\theta u$ is ultimate rotation capacity of the member; $\theta_{r}$ is recoverable rotation when member is unloading; $M_{y}$ is yield moment; $E_{h}$ is dissipated energy at the section and $\beta$ is a non-negative strength deteriorating parameter.

Global damage indices can be calculated by the weighting factors that are defined as the dissipated energy by members. The weighting factor is estimated by the following formula:

$\lambda_{i}=\frac{E_{i}}{\sum_{i}^{n} E_{i}}$

$i=1, \ldots, N$ 
Where, $N$ is the number of element and $E_{i}$ is the dissipated energy by a member.

In statistics, interdependency between two random variables is estimated by correlation coefficient. The Spearman's correlation coefficient measures the strength of association between two ranked variables. The following formula presents the relation of the Spearman correlation coefficient [16].

$\rho_{\text {Spearman }}=1-\frac{6 \sum D^{2}}{N\left(N^{2}-1\right)}$

Where, $D_{i}$ is the difference in the ranks given to the two variable values for each item of data. Also $N$ is the number of pairs of values $(\mathrm{X}, \mathrm{Y})$ in the data. It is necessary to notice that values of the Spearman correlation coefficient between 0 and 0.3 (0 and -0.3) show a weak positive (negative) correlation, values between 0.3 and 0.7 (-0.3 and -0.7) show a moderate positive (negative) correlation, and values between 0.7 and 1.0 (-0.7 and -1.0) show a strong positive (negative) correlation.

\section{Analytical Procedure}

In this study, six different concrete frames comprising five, six, seven, eight, nine, and ten story have been utilized for nonlinear dynamic analysis.
All of the story have height of $320 \mathrm{~cm}$ and the length of each beam is $600 \mathrm{~cm}$. The distances between each frame of structure have been chosen $600 \mathrm{~cm}$. All of them are shown in Figure 1. Details of the sections are presented in Tables 2 to 13 . Compressive strength of concrete is equal to $24 \mathrm{MPa}$ and strain at maximum strength of concrete is equal to $0.2 \%$. These frames were designed in accordance to ACI (318-02) code [18]. Also, seismic design of frames was done in accordance to UBC 97 code [19]. Seismic soil type equal $\mathrm{C}$ had been considered. The values of dead and live loads were $6.5 \mathrm{kN} / \mathrm{m}^{2}$ and 1.5 $\mathrm{kN} / \mathrm{m}^{2}$ respectively. After the reinforced concrete frames were designed, nonlinear dynamic analyses were performed to assess the seismic vulnerability. The computer program IDARC 7.0 [20] was utilized for nonlinear dynamic analyses. Hysteresis models which consider the effect of stiffness degradation and strength deterioration for beams and columns were applied. For beams and columns, grades related to stiffness degrading parameters are equal to moderate degrading and mild degrading respectively. In addition, for beams and columns, grades related to strength degrading parameters (energy controled) are equal to moderate deterorating and mild deteriorating respectively.

Table1. Earthquake Events

\begin{tabular}{|c|c|c|c|c|}
\hline Event & Country & Date & Station & Component \\
\hline Cape Mendocino & USA & $1992 / 04 / 25$ & 89156 Petrolia & PET090 \\
\hline Coalinga & USA & $1983 / 05 / 02$ & Pleasant Valley & H-PVY045 \\
\hline Coyote Lake & USA & $1979 / 08 / 06$ & 57383 Gilroy Array & G06230 \\
\hline Duzce & Turkey & 1999/11/12 & Bolu & BOL090 \\
\hline Erzincan & Turkey & $1992 / 03 / 13$ & 95 Erzincan & ERZ-NS \\
\hline Friuli & Italy & $1976 / 05 / 06$ & 8012 Tolmezzo & A-TMZ000 \\
\hline Gazli & Uzbekistan & $1976 / 05 / 17$ & 9201 Karakyr & GAZ000 \\
\hline Irpinia & Italy & $1980 / 11 / 23$ & Sturno & STU270 \\
\hline Kobe & Japan & $1995 / 01 / 16$ & 0 KJMA & КJM000 \\
\hline Landers & USA & $1992 / 06 / 28$ & 24 Lucerne & LCNOOO \\
\hline Loma Prieta & USA & $1989 / 10 / 18$ & 16 LGPC & LGP090 \\
\hline Imperial Valley & USA & $1979 / 10 / 15$ & 5054 Bonds & H-BCR230 \\
\hline Morgan Hill & USA & $1984 / 04 / 24$ & 57191 Halls Valley & HVR240 \\
\hline N. Palm Springs & USA & $1986 / 07 / 08$ & 5070 North & NPS300 \\
\hline Nahanni & Canada & $1985 / 12 / 23$ & 6097 Site 1 & $\mathrm{~S} 1280$ \\
\hline Northridge & USA & $1994 / 01 / 17$ & 77 Rinaldi & RRS228 \\
\hline Parkfield & USA & $1966 / 06 / 28$ & 1013 Cholame & $\mathrm{C} 02065$ \\
\hline Taiwan & Taiwan & $1986 / 11 / 14$ & 63 SMART1 O02 & $45 \mathrm{O} 02 \mathrm{NS}$ \\
\hline Victoria & Mexico & 1980/06/09 & 6604 Cerro Prieto & CPE045 \\
\hline Avaj & Iran & $2002 / 06 / 22$ & Avaj & N-E \\
\hline Bam & Iran & $2003 / 12 / 26$ & Bam & $\mathrm{L}-\mathrm{T}$ \\
\hline Manjil & Iran & $1990 / 06 / 20$ & Abbar & Transverse \\
\hline Varzaqan & Iran & $2012 / 08 / 11$ & Varzaqan & N-E \\
\hline Chi-Chi & Taiwan & 1999/09/20 & CHY006 & CHY006-E \\
\hline Tabas & Iran & $1978 / 09 / 16$ & Tabas & Transverse \\
\hline Firozabad & Iran & $2004 / 05 / 28$ & Hassankeyf & N-E \\
\hline Karebas & Iran & 1999/05/06 & Balaadeh & N-E \\
\hline Sarein & Iran & $1997 / 02 / 28$ & Kariq & L-T \\
\hline Chalfant Valley & USA & $1986 / 07 / 20$ & 54428 Zack & 270 \\
\hline Mammoth Lakes & USA & $1980 / 05 / 25$ & 54099 Convict & CVK180 \\
\hline
\end{tabular}



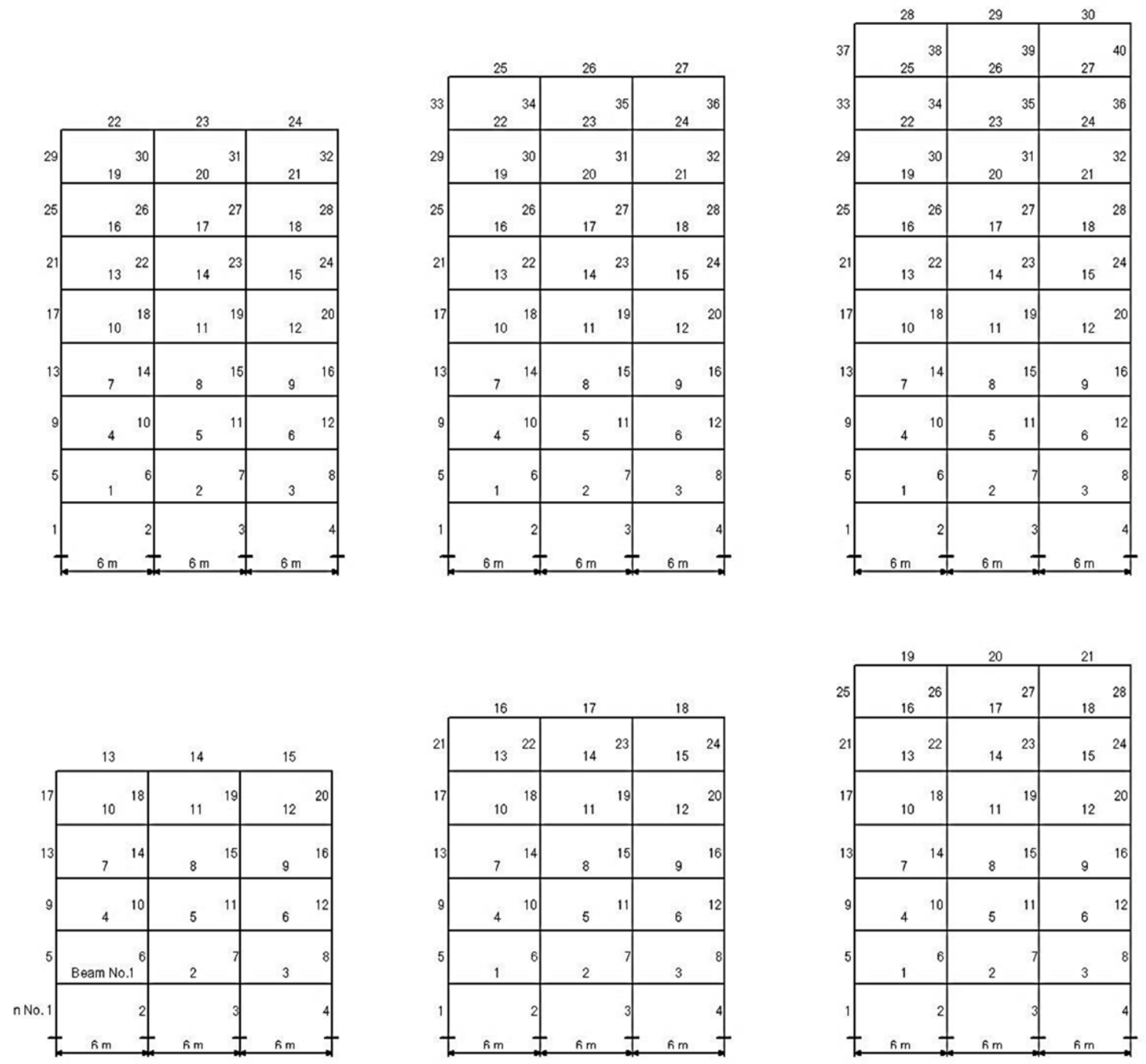

Figure 1. Reinforced Concrete Frames

Table 2. Details of the Five-story Frame Beams

\begin{tabular}{ccccccccc}
\hline No. & $\begin{array}{c}\text { Section } \\
\text { Dimensions }\end{array}$ & $\begin{array}{c}\text { Maximum } \\
\text { Rebar } \\
\text { Percentage }\end{array}$ & No. & $\begin{array}{c}\text { Section } \\
\text { Dimensions }\end{array}$ & $\begin{array}{c}\text { Maximum Rebar } \\
\text { Percentage }\end{array}$ & No. & $\begin{array}{c}\text { Section } \\
\text { Dimensions }\end{array}$ & $\begin{array}{c}\text { Maximum } \\
\text { Rebar } \\
\text { Percentage }\end{array}$ \\
\hline 1 & $50 \times 40 \mathrm{~cm}$ & $1.09 \%$ & 6 & $50 \times 40 \mathrm{~cm}$ & $1.24 \%$ & 11 & $45 \times 40 \mathrm{~cm}$ & $0.96 \%$ \\
2 & $50 \times 40 \mathrm{~cm}$ & $1.06 \%$ & 7 & $45 \times 40 \mathrm{~cm}$ & $1.21 \%$ & 12 & $45 \times 40 \mathrm{~cm}$ & $0.98 \%$ \\
3 & $50 \times 40 \mathrm{~cm}$ & $1.09 \%$ & 8 & $45 \times 40 \mathrm{~cm}$ & $1.18 \%$ & 13 & $45 \times 40 \mathrm{~cm}$ & $0.75 \%$ \\
4 & $50 \times 40 \mathrm{~cm}$ & $1.24 \%$ & 9 & $45 \times 40 \mathrm{~cm}$ & $1.21 \%$ & 14 & $45 \times 40 \mathrm{~cm}$ & $0.73 \%$ \\
5 & $50 \times 40 \mathrm{~cm}$ & $1.21 \%$ & 10 & $45 \times 40 \mathrm{~cm}$ & $0.98 \%$ & 15 & $45 \times 40 \mathrm{~cm}$ & $0.75 \%$ \\
\hline
\end{tabular}

Table 3. Details of the Five-story Frame Columns

\begin{tabular}{ccccccccc}
\hline No. & $\begin{array}{c}\text { Section } \\
\text { Dimensions }\end{array}$ & $\begin{array}{c}\text { Maximum } \\
\text { Rebar } \\
\text { Percentage }\end{array}$ & No. & $\begin{array}{c}\text { Section } \\
\text { Dimensions }\end{array}$ & $\begin{array}{c}\text { Maximum Rebar } \\
\text { Percentage }\end{array}$ & No. & $\begin{array}{c}\text { Section } \\
\text { Dimensions }\end{array}$ & $\begin{array}{c}\text { Maximum } \\
\text { Rebar } \\
\text { Percentage }\end{array}$ \\
\hline 1 & $50 \times 50 \mathrm{~cm}$ & $2.9 \%$ & 8 & $50 \times 50 \mathrm{~cm}$ & $2.90 \%$ & 15 & $45 \times 45 \mathrm{~cm}$ & $1.24 \%$ \\
2 & $50 \times 50 \mathrm{~cm}$ & $2.9 \%$ & 9 & $50 \times 50 \mathrm{~cm}$ & $1.24 \%$ & 16 & $45 \times 45 \mathrm{~cm}$ & $1.24 \%$ \\
3 & $50 \times 50 \mathrm{~cm}$ & $2.9 \%$ & 10 & $50 \times 50 \mathrm{~cm}$ & $1.24 \%$ & 17 & $45 \times 45 \mathrm{~cm}$ & $1.24 \%$ \\
4 & $50 \times 50 \mathrm{~cm}$ & $2.9 \%$ & 11 & $50 \times 50 \mathrm{~cm}$ & $1.24 \%$ & 18 & $45 \times 45 \mathrm{~cm}$ & $1.24 \%$ \\
5 & $50 \times 50 \mathrm{~cm}$ & $2.9 \%$ & 12 & $50 \times 50 \mathrm{~cm}$ & $1.24 \%$ & 19 & $45 \times 45 \mathrm{~cm}$ & $1.24 \%$ \\
6 & $50 \times 50 \mathrm{~cm}$ & $2.9 \%$ & 13 & $45 \times 45 \mathrm{~cm}$ & $1.24 \%$ & 20 & $45 \times 45 \mathrm{~cm}$ & $1.24 \%$ \\
7 & $50 \times 50 \mathrm{~cm}$ & $2.9 \%$ & 14 & $45 \times 45 \mathrm{~cm}$ & $1.24 \%$ & 15 & $45 \times 45 \mathrm{~cm}$ & $1.24 \%$ \\
\hline
\end{tabular}


Table 4. Details of the Six-story Frame Beams

\begin{tabular}{ccccccccc}
\hline No. & $\begin{array}{c}\text { Section } \\
\text { Dimensions }\end{array}$ & $\begin{array}{c}\text { Maximum } \\
\text { Rebar } \\
\text { Percentage }\end{array}$ & No. & $\begin{array}{c}\text { Section } \\
\text { Dimensions }\end{array}$ & $\begin{array}{c}\text { Maximum Rebar } \\
\text { Percentage }\end{array}$ & No. & $\begin{array}{c}\text { Section } \\
\text { Dimensions }\end{array}$ & $\begin{array}{c}\text { Maximum } \\
\text { Rebar } \\
\text { Percentage }\end{array}$ \\
\hline 1 & $55 \times 40 \mathrm{~cm}$ & $2.99 \%$ & 7 & $50 \times 40 \mathrm{~cm}$ & $1.45 \%$ & 13 & $50 \times 40 \mathrm{~cm}$ & $1.45 \%$ \\
2 & $55 \times 40 \mathrm{~cm}$ & $2.99 \%$ & 8 & $50 \times 40 \mathrm{~cm}$ & $1.45 \%$ & 14 & $50 \times 40 \mathrm{~cm}$ & $1.45 \%$ \\
3 & $55 \times 40 \mathrm{~cm}$ & $2.99 \%$ & 9 & $50 \times 40 \mathrm{~cm}$ & $1.45 \%$ & 15 & $50 \times 40 \mathrm{~cm}$ & $1.45 \%$ \\
4 & $55 \times 40 \mathrm{~cm}$ & $2.99 \%$ & 10 & $50 \times 40 \mathrm{~cm}$ & $1.45 \%$ & 16 & $50 \times 40 \mathrm{~cm}$ & $1.45 \%$ \\
5 & $55 \times 40 \mathrm{~cm}$ & $2.99 \%$ & 11 & $50 \times 40 \mathrm{~cm}$ & $1.45 \%$ & 17 & $50 \times 40 \mathrm{~cm}$ & $1.45 \%$ \\
6 & $55 \times 40 \mathrm{~cm}$ & $2.99 \%$ & 12 & $50 \times 40 \mathrm{~cm}$ & $1.45 \%$ & 18 & $50 \times 40 \mathrm{~cm}$ & $1.45 \%$ \\
\hline
\end{tabular}

Table 5. Details of the Six-story Frame Columns

\begin{tabular}{|c|c|c|c|c|c|c|c|c|}
\hline No. & $\begin{array}{c}\text { Section } \\
\text { Dimensions }\end{array}$ & $\begin{array}{c}\text { Maximum } \\
\text { Rebar } \\
\text { Percentage }\end{array}$ & No. & $\begin{array}{c}\text { Section } \\
\text { Dimensions }\end{array}$ & $\begin{array}{c}\text { Maximum Rebar } \\
\text { Percentage }\end{array}$ & No. & $\begin{array}{c}\text { Section } \\
\text { Dimensions }\end{array}$ & $\begin{array}{c}\text { Maximum } \\
\text { Rebar } \\
\text { Percentage }\end{array}$ \\
\hline 1 & $55 \times 55 \mathrm{~cm}$ & $2.9 \%$ & 9 & $50 \times 50 \mathrm{~cm}$ & $1.45 \%$ & 17 & $50 \times 50 \mathrm{~cm}$ & $1.45 \%$ \\
\hline 2 & $55 \times 55 \mathrm{~cm}$ & $2.9 \%$ & 10 & $50 \times 50 \mathrm{~cm}$ & $1.45 \%$ & 18 & $50 \times 50 \mathrm{~cm}$ & $1.45 \%$ \\
\hline 3 & $55 \times 55 \mathrm{~cm}$ & $2.9 \%$ & 11 & $50 \times 50 \mathrm{~cm}$ & $1.45 \%$ & 19 & $50 \times 50 \mathrm{~cm}$ & $1.45 \%$ \\
\hline 4 & $55 \times 55 \mathrm{~cm}$ & $2.9 \%$ & 12 & $50 \times 50 \mathrm{~cm}$ & $1.45 \%$ & 20 & $50 \times 50 \mathrm{~cm}$ & $1.45 \%$ \\
\hline 5 & $55 \times 55 \mathrm{~cm}$ & $2.9 \%$ & 13 & $50 \times 50 \mathrm{~cm}$ & $1.45 \%$ & 21 & $50 \times 50 \mathrm{~cm}$ & $1.45 \%$ \\
\hline 6 & $55 \times 55 \mathrm{~cm}$ & $2.9 \%$ & 14 & $50 \times 50 \mathrm{~cm}$ & $1.45 \%$ & 22 & $50 \times 50 \mathrm{~cm}$ & $1.45 \%$ \\
\hline 7 & $55 \times 55 \mathrm{~cm}$ & $2.9 \%$ & 15 & $50 \times 50 \mathrm{~cm}$ & $1.45 \%$ & 23 & $50 \times 50 \mathrm{~cm}$ & $1.45 \%$ \\
\hline 8 & $55 \times 55 \mathrm{~cm}$ & $2.9 \%$ & 16 & $50 \times 50 \mathrm{~cm}$ & $1.45 \%$ & 24 & $50 \times 50 \mathrm{~cm}$ & $1.45 \%$ \\
\hline
\end{tabular}

Table 6. Details of the Seven-story Frame Beams

\begin{tabular}{|c|c|c|c|c|c|c|c|c|}
\hline No. & $\begin{array}{c}\text { Section } \\
\text { Dimensions }\end{array}$ & $\begin{array}{l}\text { Maximum } \\
\text { Rebar } \\
\text { Percentage }\end{array}$ & No. & $\begin{array}{c}\text { Section } \\
\text { Dimensions }\end{array}$ & $\begin{array}{c}\text { Maximum Rebar } \\
\text { Percentage }\end{array}$ & No. & $\begin{array}{c}\text { Section } \\
\text { Dimensions }\end{array}$ & $\begin{array}{c}\text { Maximum } \\
\text { Rebar } \\
\text { Percentage }\end{array}$ \\
\hline 1 & $60 \times 40 \mathrm{~cm}$ & $1.00 \%$ & 8 & $60 \times 40 \mathrm{~cm}$ & $1.26 \%$ & 15 & $55 \times 40 \mathrm{~cm}$ & $1.02 \%$ \\
\hline 2 & $60 \times 40 \mathrm{~cm}$ & $1.00 \%$ & 9 & $60 \times 40 \mathrm{~cm}$ & $1.30 \%$ & 16 & $55 \times 40 \mathrm{~cm}$ & $0.83 \%$ \\
\hline 3 & $60 \times 40 \mathrm{~cm}$ & $1.00 \%$ & 10 & $55 \times 40 \mathrm{~cm}$ & $1.20 \%$ & 17 & $55 \times 40 \mathrm{~cm}$ & $0.80 \%$ \\
\hline 4 & $60 \times 40 \mathrm{~cm}$ & $1.26 \%$ & 11 & $55 \times 40 \mathrm{~cm}$ & $1.16 \%$ & 18 & $55 \times 40 \mathrm{~cm}$ & $0.83 \%$ \\
\hline 5 & $60 \times 40 \mathrm{~cm}$ & $1.24 \%$ & 12 & $55 \times 40 \mathrm{~cm}$ & $1.20 \%$ & 19 & $55 \times 40 \mathrm{~cm}$ & $0.63 \%$ \\
\hline 6 & $60 \times 40 \mathrm{~cm}$ & $1.26 \%$ & 13 & $55 \times 40 \mathrm{~cm}$ & $1.02 \%$ & 20 & $55 \times 40 \mathrm{~cm}$ & $0.63 \%$ \\
\hline 7 & $60 \times 40 \mathrm{~cm}$ & $1.30 \%$ & 14 & $55 \times 40 \mathrm{~cm}$ & $0.99 \%$ & 21 & $55 \times 40 \mathrm{~cm}$ & $0.63 \%$ \\
\hline
\end{tabular}

Table 7. Details of the Seven-story Frame Columns

\begin{tabular}{|c|c|c|c|c|c|c|c|c|}
\hline No. & $\begin{array}{c}\text { Section } \\
\text { Dimensions }\end{array}$ & $\begin{array}{l}\text { Maximum } \\
\text { Rebar } \\
\text { Percentage }\end{array}$ & No. & $\begin{array}{c}\text { Section } \\
\text { Dimensions }\end{array}$ & $\begin{array}{l}\text { Maximum Rebar } \\
\text { Percentage }\end{array}$ & No. & $\begin{array}{c}\text { Section } \\
\text { Dimensions }\end{array}$ & $\begin{array}{c}\text { Maximum } \\
\text { Rebar } \\
\text { Percentage }\end{array}$ \\
\hline 1 & $60 \times 60 \mathrm{~cm}$ & $2.09 \%$ & 11 & $60 \times 60 \mathrm{~cm}$ & $2.09 \%$ & 21 & $55 \times 55 \mathrm{~cm}$ & $1.25 \%$ \\
\hline 2 & $60 \times 60 \mathrm{~cm}$ & $2.09 \%$ & 12 & $60 \times 60 \mathrm{~cm}$ & $2.09 \%$ & 22 & $55 \times 55 \mathrm{~cm}$ & $1.25 \%$ \\
\hline 3 & $60 \times 60 \mathrm{~cm}$ & $2.09 \%$ & 13 & $55 \times 55 \mathrm{~cm}$ & $1.25 \%$ & 23 & $55 \times 55 \mathrm{~cm}$ & $1.25 \%$ \\
\hline 4 & $60 \times 60 \mathrm{~cm}$ & $2.09 \%$ & 14 & $55 \times 55 \mathrm{~cm}$ & $1.25 \%$ & 24 & $55 \times 55 \mathrm{~cm}$ & $1.25 \%$ \\
\hline 5 & $60 \times 60 \mathrm{~cm}$ & $2.09 \%$ & 15 & $55 \times 55 \mathrm{~cm}$ & $1.25 \%$ & 25 & $55 \times 55 \mathrm{~cm}$ & $1.25 \%$ \\
\hline 6 & $60 \times 60 \mathrm{~cm}$ & $2.09 \%$ & 16 & $55 \times 55 \mathrm{~cm}$ & $1.25 \%$ & 26 & $55 \times 55 \mathrm{~cm}$ & $1.25 \%$ \\
\hline 7 & $60 \times 60 \mathrm{~cm}$ & $2.09 \%$ & 17 & $55 \times 55 \mathrm{~cm}$ & $1.25 \%$ & 27 & $55 \times 55 \mathrm{~cm}$ & $1.25 \%$ \\
\hline 8 & $60 \times 60 \mathrm{~cm}$ & $2.09 \%$ & 18 & $55 \times 55 \mathrm{~cm}$ & $1.25 \%$ & 28 & $55 \times 55 \mathrm{~cm}$ & $1.25 \%$ \\
\hline 9 & $60 \times 60 \mathrm{~cm}$ & $2.09 \%$ & 19 & $55 \times 55 \mathrm{~cm}$ & $1.25 \%$ & - & - & - \\
\hline 10 & $60 \times 60 \mathrm{~cm}$ & $2.09 \%$ & 20 & $55 \times 55 \mathrm{~cm}$ & $1.25 \%$ & - & - & - \\
\hline
\end{tabular}

Table 8. Details of the Eight-story Frame Beams

\begin{tabular}{|c|c|c|c|c|c|c|c|c|}
\hline No. & $\begin{array}{c}\text { Section } \\
\text { Dimensions }\end{array}$ & $\begin{array}{c}\text { Maximum } \\
\text { Rebar } \\
\text { Percentage }\end{array}$ & No. & $\begin{array}{c}\text { Section } \\
\text { Dimensions }\end{array}$ & $\begin{array}{c}\text { Maximum Rebar } \\
\text { Percentage }\end{array}$ & No. & $\begin{array}{c}\text { Section } \\
\text { Dimensions }\end{array}$ & $\begin{array}{c}\text { Maximum } \\
\text { Rebar } \\
\text { Percentage }\end{array}$ \\
\hline 1 & $60 \times 40 \mathrm{~cm}$ & $1.03 \%$ & 9 & $60 \times 40 \mathrm{~cm}$ & $1.44 \%$ & 17 & $50 \times 40 \mathrm{~cm}$ & $1.12 \%$ \\
\hline 2 & $60 \times 40 \mathrm{~cm}$ & $1.02 \%$ & 10 & $55 \times 40 \mathrm{~cm}$ & $1.41 \%$ & 18 & $50 \times 40 \mathrm{~cm}$ & $1.15 \%$ \\
\hline 3 & $60 \times 40 \mathrm{~cm}$ & $1.03 \%$ & 11 & $55 \times 40 \mathrm{~cm}$ & $1.37 \%$ & 19 & $50 \times 40 \mathrm{~cm}$ & $0.94 \%$ \\
\hline 4 & $60 \times 40 \mathrm{~cm}$ & $1.36 \%$ & 12 & $55 \times 40 \mathrm{~cm}$ & $1.41 \%$ & 20 & $50 \times 40 \mathrm{~cm}$ & $0.90 \%$ \\
\hline 5 & $60 \times 40 \mathrm{~cm}$ & $1.33 \%$ & 13 & $55 \times 40 \mathrm{~cm}$ & $1.28 \%$ & 21 & $50 \times 40 \mathrm{~cm}$ & $0.94 \%$ \\
\hline 6 & $60 \times 40 \mathrm{~cm}$ & $1.36 \%$ & 14 & $55 \times 40 \mathrm{~cm}$ & $1.24 \%$ & 22 & $50 \times 40 \mathrm{~cm}$ & $0.73 \%$ \\
\hline 7 & $60 \times 40 \mathrm{~cm}$ & $1.44 \%$ & 15 & $55 \times 40 \mathrm{~cm}$ & $1.28 \%$ & 23 & $50 \times 40 \mathrm{~cm}$ & $0.75 \%$ \\
\hline 8 & $60 \times 40 \mathrm{~cm}$ & $1.41 \%$ & 16 & $50 \times 40 \mathrm{~cm}$ & $1.15 \%$ & 24 & $50 \times 40 \mathrm{~cm}$ & $0.73 \%$ \\
\hline
\end{tabular}


Table 9. Details of the Eight-story Frame Columns

\begin{tabular}{ccccccccc}
\hline No. & $\begin{array}{c}\text { Section } \\
\text { Dimensions }\end{array}$ & $\begin{array}{c}\text { Maximum } \\
\text { Rebar } \\
\text { Percentage }\end{array}$ & No. & $\begin{array}{c}\text { Section } \\
\text { Dimensions }\end{array}$ & $\begin{array}{c}\text { Maximum Rebar } \\
\text { Percentage }\end{array}$ & No. & $\begin{array}{c}\text { Section } \\
\text { Dimensions }\end{array}$ & $\begin{array}{c}\text { Maximum } \\
\text { Rebar } \\
\text { Percentage }\end{array}$ \\
\hline 1 & $60 \times 60 \mathrm{~cm}$ & $2.31 \%$ & 12 & $60 \times 60 \mathrm{~cm}$ & $2.31 \%$ & 23 & $50 \times 50 \mathrm{~cm}$ & $1.45 \%$ \\
2 & $60 \times 60 \mathrm{~cm}$ & $2.31 \%$ & 13 & $55 \times 55 \mathrm{~cm}$ & $1.79 \%$ & 24 & $50 \times 50 \mathrm{~cm}$ & $1.45 \%$ \\
3 & $60 \times 60 \mathrm{~cm}$ & $2.31 \%$ & 14 & $55 \times 55 \mathrm{~cm}$ & $1.79 \%$ & 25 & $50 \times 50 \mathrm{~cm}$ & $1.45 \%$ \\
4 & $60 \times 60 \mathrm{~cm}$ & $2.31 \%$ & 15 & $55 \times 55 \mathrm{~cm}$ & $1.79 \%$ & 26 & $50 \times 50 \mathrm{~cm}$ & $1.45 \%$ \\
5 & $60 \times 60 \mathrm{~cm}$ & $2.31 \%$ & 16 & $55 \times 55 \mathrm{~cm}$ & $1.79 \%$ & 27 & $50 \times 50 \mathrm{~cm}$ & $1.45 \%$ \\
6 & $60 \times 60 \mathrm{~cm}$ & $2.31 \%$ & 17 & $55 \times 55 \mathrm{~cm}$ & $1.79 \%$ & 28 & $50 \times 50 \mathrm{~cm}$ & $1.45 \%$ \\
7 & $60 \times 60 \mathrm{~cm}$ & $2.31 \%$ & 18 & $55 \times 55 \mathrm{~cm}$ & $1.79 \%$ & 29 & $50 \times 50 \mathrm{~cm}$ & $1.45 \%$ \\
8 & $60 \times 60 \mathrm{~cm}$ & $2.31 \%$ & 19 & $55 \times 55 \mathrm{~cm}$ & $1.79 \%$ & 30 & $50 \times 50 \mathrm{~cm}$ & $1.45 \%$ \\
9 & $60 \times 60 \mathrm{~cm}$ & $2.31 \%$ & 20 & $55 \times 55 \mathrm{~cm}$ & $1.79 \%$ & 31 & $50 \times 50 \mathrm{~cm}$ & $1.45 \%$ \\
10 & $60 \times 60 \mathrm{~cm}$ & $2.31 \%$ & 21 & $50 \times 50 \mathrm{~cm}$ & $1.45 \%$ & 32 & $50 \times 50 \mathrm{~cm}$ & $1.45 \%$ \\
11 & $60 \times 60 \mathrm{~cm}$ & $2.31 \%$ & 22 & $50 \times 50 \mathrm{~cm}$ & $1.45 \%$ & - & - & - \\
\hline
\end{tabular}

Table 10. Details of the Nine-story Frame Beams

\begin{tabular}{ccccccccc}
\hline No. & $\begin{array}{c}\text { Section } \\
\text { Dimensions }\end{array}$ & $\begin{array}{c}\text { Maximum } \\
\text { Rebar } \\
\text { Percentage }\end{array}$ & No. & $\begin{array}{c}\text { Section } \\
\text { Dimensions }\end{array}$ & $\begin{array}{c}\text { Maximum Rebar } \\
\text { Percentage }\end{array}$ & No. & $\begin{array}{c}\text { Section } \\
\text { Dimensions }\end{array}$ & $\begin{array}{c}\text { Maximum } \\
\text { Rebar } \\
\text { Percentage }\end{array}$ \\
\hline 1 & $60 \times 40 \mathrm{~cm}$ & $1.05 \%$ & 10 & $55 \times 40 \mathrm{~cm}$ & $1.39 \%$ & 19 & $50 \times 40 \mathrm{~cm}$ & $0.90 \%$ \\
2 & $60 \times 40 \mathrm{~cm}$ & $1.03 \%$ & 11 & $55 \times 40 \mathrm{~cm}$ & $1.34 \%$ & 20 & $50 \times 40 \mathrm{~cm}$ & $0.86 \%$ \\
3 & $60 \times 40 \mathrm{~cm}$ & $1.05 \%$ & 12 & $55 \times 40 \mathrm{~cm}$ & $1.39 \%$ & 21 & $50 \times 40 \mathrm{~cm}$ & $0.90 \%$ \\
4 & $60 \times 40 \mathrm{~cm}$ & $1.37 \%$ & 13 & $55 \times 40 \mathrm{~cm}$ & $1.26 \%$ & 22 & $50 \times 40 \mathrm{~cm}$ & $0.68 \%$ \\
5 & $60 \times 40 \mathrm{~cm}$ & $1.33 \%$ & 14 & $55 \times 40 \mathrm{~cm}$ & $1.20 \%$ & 23 & $50 \times 40 \mathrm{~cm}$ & $0.64 \%$ \\
6 & $60 \times 40 \mathrm{~cm}$ & $1.37 \%$ & 15 & $55 \times 40 \mathrm{~cm}$ & $1.26 \%$ & 24 & $50 \times 40 \mathrm{~cm}$ & $0.68 \%$ \\
7 & $60 \times 40 \mathrm{~cm}$ & $1.43 \%$ & 16 & $55 \times 40 \mathrm{~cm}$ & $1.13 \%$ & 25 & $50 \times 40 \mathrm{~cm}$ & $0.52 \%$ \\
8 & $60 \times 40 \mathrm{~cm}$ & $1.39 \%$ & 17 & $55 \times 40 \mathrm{~cm}$ & $1.08 \%$ & 26 & $50 \times 40 \mathrm{~cm}$ & $0.55 \%$ \\
9 & $60 \times 40 \mathrm{~cm}$ & $1.43 \%$ & 18 & $55 \times 40 \mathrm{~cm}$ & $1.13 \%$ & 27 & $50 \times 40 \mathrm{~cm}$ & $0.52 \%$ \\
\hline
\end{tabular}

Table 11. Details of the Nine-story Frame Columns

\begin{tabular}{ccccccccc}
\hline No. & $\begin{array}{c}\text { Section } \\
\text { Dimensions }\end{array}$ & $\begin{array}{c}\text { Maximum } \\
\text { Rebar } \\
\text { Percentage }\end{array}$ & No. & $\begin{array}{c}\text { Section } \\
\text { Dimensions }\end{array}$ & $\begin{array}{c}\text { Maximum Rebar } \\
\text { Percentage }\end{array}$ & No. & $\begin{array}{c}\text { Section } \\
\text { Dimensions }\end{array}$ & $\begin{array}{c}\text { Maximum } \\
\text { Rebar } \\
\text { Percentage }\end{array}$ \\
\hline 1 & $60 \times 60 \mathrm{~cm}$ & $3.01 \%$ & 13 & $55 \times 55 \mathrm{~cm}$ & $1.79 \%$ & 25 & $50 \times 50 \mathrm{~cm}$ & $1.45 \%$ \\
2 & $60 \times 60 \mathrm{~cm}$ & $3.01 \%$ & 14 & $55 \times 55 \mathrm{~cm}$ & $1.79 \%$ & 26 & $50 \times 50 \mathrm{~cm}$ & $1.45 \%$ \\
3 & $60 \times 60 \mathrm{~cm}$ & $3.01 \%$ & 15 & $55 \times 55 \mathrm{~cm}$ & $1.79 \%$ & 27 & $50 \times 50 \mathrm{~cm}$ & $1.45 \%$ \\
4 & $60 \times 60 \mathrm{~cm}$ & $3.01 \%$ & 16 & $55 \times 55 \mathrm{~cm}$ & $1.79 \%$ & 28 & $50 \times 50 \mathrm{~cm}$ & $1.45 \%$ \\
5 & $60 \times 60 \mathrm{~cm}$ & $3.01 \%$ & 17 & $55 \times 55 \mathrm{~cm}$ & $1.79 \%$ & 29 & $50 \times 50 \mathrm{~cm}$ & $1.45 \%$ \\
6 & $60 \times 60 \mathrm{~cm}$ & $3.01 \%$ & 18 & $55 \times 55 \mathrm{~cm}$ & $1.79 \%$ & 30 & $50 \times 50 \mathrm{~cm}$ & $1.45 \%$ \\
7 & $60 \times 60 \mathrm{~cm}$ & $3.01 \%$ & 19 & $55 \times 55 \mathrm{~cm}$ & $1.79 \%$ & 31 & $50 \times 50 \mathrm{~cm}$ & $1.45 \%$ \\
8 & $60 \times 60 \mathrm{~cm}$ & $3.01 \%$ & 20 & $55 \times 55 \mathrm{~cm}$ & $1.79 \%$ & 32 & $50 \times 50 \mathrm{~cm}$ & $1.45 \%$ \\
9 & $60 \times 60 \mathrm{~cm}$ & $3.01 \%$ & 21 & $50 \times 50 \mathrm{~cm}$ & $1.45 \%$ & 33 & $50 \times 50 \mathrm{~cm}$ & $1.45 \%$ \\
10 & $60 \times 60 \mathrm{~cm}$ & $3.01 \%$ & 22 & $50 \times 50 \mathrm{~cm}$ & $1.45 \%$ & 34 & $50 \times 50 \mathrm{~cm}$ & $1.45 \%$ \\
11 & $60 \times 60 \mathrm{~cm}$ & $3.01 \%$ & 23 & $50 \times 50 \mathrm{~cm}$ & $1.45 \%$ & 35 & $50 \times 50 \mathrm{~cm}$ & $1.45 \%$ \\
12 & $60 \times 60 \mathrm{~cm}$ & $3.01 \%$ & 24 & $50 \times 50 \mathrm{~cm}$ & $1.45 \%$ & 36 & $50 \times 50 \mathrm{~cm}$ & $1.45 \%$ \\
\hline
\end{tabular}

Table 12. Details of the Ten-story Frame Beams

\begin{tabular}{|c|c|c|c|c|c|c|c|c|}
\hline No. & $\begin{array}{c}\text { Section } \\
\text { Dimensions }\end{array}$ & $\begin{array}{c}\text { Maximum } \\
\text { Rebar } \\
\text { Percentage }\end{array}$ & No. & $\begin{array}{c}\text { Section } \\
\text { Dimensions }\end{array}$ & $\begin{array}{c}\text { Maximum Rebar } \\
\text { Percentage }\end{array}$ & No. & $\begin{array}{c}\text { Section } \\
\text { Dimensions }\end{array}$ & $\begin{array}{c}\text { Maximum } \\
\text { Rebar } \\
\text { Percentage }\end{array}$ \\
\hline 1 & $0.98 \%$ & $65 \times 40 \mathrm{~cm}$ & 11 & $1.57 \%$ & $60 \times 40 \mathrm{~cm}$ & 21 & $60 \times 40 \mathrm{~cm}$ & $1.29 \%$ \\
\hline 2 & $0.96 \%$ & $65 \times 40 \mathrm{~cm}$ & 12 & $1.61 \%$ & $60 \times 40 \mathrm{~cm}$ & 22 & $55 \times 40 \mathrm{~cm}$ & $1.15 \%$ \\
\hline 3 & $0.98 \%$ & $65 \times 40 \mathrm{~cm}$ & 13 & $1.56 \%$ & $60 \times 40 \mathrm{~cm}$ & 23 & $55 \times 40 \mathrm{~cm}$ & $1.10 \%$ \\
\hline 4 & $1.33 \%$ & $65 \times 40 \mathrm{~cm}$ & 14 & $1.52 \%$ & $60 \times 40 \mathrm{~cm}$ & 24 & $55 \times 40 \mathrm{~cm}$ & $1.15 \%$ \\
\hline 5 & $1.31 \%$ & $65 \times 40 \mathrm{~cm}$ & 15 & $1.56 \%$ & $60 \times 40 \mathrm{~cm}$ & 25 & $55 \times 40 \mathrm{~cm}$ & $0.95 \%$ \\
\hline 6 & $1.33 \%$ & $65 \times 40 \mathrm{~cm}$ & 16 & $1.44 \%$ & $60 \times 40 \mathrm{~cm}$ & 26 & $55 \times 40 \mathrm{~cm}$ & $0.91 \%$ \\
\hline 7 & $1.47 \%$ & $65 \times 40 \mathrm{~cm}$ & 17 & $1.40 \%$ & $60 \times 40 \mathrm{~cm}$ & 27 & $55 \times 40 \mathrm{~cm}$ & $0.95 \%$ \\
\hline 8 & $1.43 \%$ & $65 \times 40 \mathrm{~cm}$ & 18 & $1.44 \%$ & $60 \times 40 \mathrm{~cm}$ & 28 & $55 \times 40 \mathrm{~cm}$ & $0.77 \%$ \\
\hline 9 & $1.47 \%$ & $65 \times 40 \mathrm{~cm}$ & 19 & $1.29 \%$ & $60 \times 40 \mathrm{~cm}$ & 29 & $55 \times 40 \mathrm{~cm}$ & $0.77 \%$ \\
\hline 10 & $1.61 \%$ & $60 \times 40 \mathrm{~cm}$ & 20 & $1.24 \%$ & $60 \times 40 \mathrm{~cm}$ & 30 & $55 \times 40 \mathrm{~cm}$ & $0.77 \%$ \\
\hline
\end{tabular}


Table 13. Details of the Ten-story Frame Columns

\begin{tabular}{ccccccccc}
\hline No. & $\begin{array}{c}\text { Section } \\
\text { Dimensions }\end{array}$ & $\begin{array}{c}\text { Maximum } \\
\text { Rebar } \\
\text { Percentage }\end{array}$ & No. & $\begin{array}{c}\text { Section } \\
\text { Dimensions }\end{array}$ & $\begin{array}{c}\text { Maximum Rebar } \\
\text { Percentage }\end{array}$ & No. & $\begin{array}{c}\text { Section } \\
\text { Dimensions }\end{array}$ & $\begin{array}{c}\text { Maximum } \\
\text { Rebar } \\
\text { Percentage }\end{array}$ \\
\hline 1 & $3.50 \%$ & $65 \times 65 \mathrm{~cm}$ & 15 & $2.05 \%$ & $60 \times 60 \mathrm{~cm}$ & 29 & $55 \times 55 \mathrm{~cm}$ & $1.63 \%$ \\
2 & $3.50 \%$ & $65 \times 65 \mathrm{~cm}$ & 16 & $2.05 \%$ & $60 \times 60 \mathrm{~cm}$ & 30 & $55 \times 55 \mathrm{~cm}$ & $1.63 \%$ \\
3 & $3.50 \%$ & $65 \times 65 \mathrm{~cm}$ & 17 & $2.05 \%$ & $60 \times 60 \mathrm{~cm}$ & 31 & $55 \times 55 \mathrm{~cm}$ & $1.63 \%$ \\
4 & $3.50 \%$ & $65 \times 65 \mathrm{~cm}$ & 18 & $2.05 \%$ & $60 \times 60 \mathrm{~cm}$ & 32 & $55 \times 55 \mathrm{~cm}$ & $1.63 \%$ \\
5 & $3.50 \%$ & $65 \times 65 \mathrm{~cm}$ & 19 & $2.05 \%$ & $60 \times 60 \mathrm{~cm}$ & 33 & $55 \times 55 \mathrm{~cm}$ & $1.63 \%$ \\
6 & $3.50 \%$ & $65 \times 65 \mathrm{~cm}$ & 20 & $2.05 \%$ & $60 \times 60 \mathrm{~cm}$ & 34 & $55 \times 55 \mathrm{~cm}$ & $1.63 \%$ \\
7 & $3.50 \%$ & $65 \times 65 \mathrm{~cm}$ & 21 & $2.05 \%$ & $60 \times 60 \mathrm{~cm}$ & 35 & $55 \times 55 \mathrm{~cm}$ & $1.63 \%$ \\
8 & $3.50 \%$ & $65 \times 65 \mathrm{~cm}$ & 22 & $2.05 \%$ & $60 \times 60 \mathrm{~cm}$ & 36 & $55 \times 55 \mathrm{~cm}$ & $1.63 \%$ \\
9 & $3.50 \%$ & $65 \times 65 \mathrm{~cm}$ & 23 & $2.05 \%$ & $60 \times 60 \mathrm{~cm}$ & 37 & $55 \times 55 \mathrm{~cm}$ & $1.63 \%$ \\
10 & $3.50 \%$ & $65 \times 65 \mathrm{~cm}$ & 24 & $2.05 \%$ & $60 \times 60 \mathrm{~cm}$ & 38 & $55 \times 55 \mathrm{~cm}$ & $1.63 \%$ \\
11 & $3.50 \%$ & $65 \times 65 \mathrm{~cm}$ & 25 & $2.05 \%$ & $60 \times 60 \mathrm{~cm}$ & 39 & $55 \times 55 \mathrm{~cm}$ & $1.63 \%$ \\
12 & $3.50 \%$ & $65 \times 65 \mathrm{~cm}$ & 26 & $2.05 \%$ & $60 \times 60 \mathrm{~cm}$ & 40 & $55 \times 55 \mathrm{~cm}$ & $1.63 \%$ \\
13 & $2.05 \%$ & $60 \times 60 \mathrm{~cm}$ & 27 & $1.63 \%$ & $60 \times 60 \mathrm{~cm}$ & - & - & - \\
14 & $2.05 \%$ & $60 \times 60 \mathrm{~cm}$ & 28 & $1.63 \%$ & $60 \times 60 \mathrm{~cm}$ & - & - & - \\
\hline
\end{tabular}

Table 14. Values of the Seismic Parameters

\begin{tabular}{|c|c|c|c|c|c|c|c|c|c|c|c|c|c|c|c|c|}
\hline Ear & $\begin{array}{c}\text { PGA } \\
\text { (g) }\end{array}$ & $\begin{array}{c}\text { PGV } \\
(\mathrm{cm} / \mathrm{sec})\end{array}$ & $\begin{array}{l}\text { PGD } \\
(\mathrm{cm})\end{array}$ & $\begin{array}{c}\mathrm{V}_{\max } / \mathrm{A}_{\text {max }} \\
(\mathrm{sec})\end{array}$ & $\begin{array}{c}\text { x ARMS } \\
\text { (g) }\end{array}$ & $\begin{array}{l}\text { VRMS } \\
(\mathrm{cm} / \mathrm{sec})\end{array}$ & $\begin{array}{c}\text { DRMS } \\
(\mathrm{cm})\end{array}$ & $\begin{array}{c}\mathrm{IA} \\
(\mathrm{m} / \mathrm{sec})\end{array}$ & $\mathrm{IC}$ & $\begin{array}{c}\text { SED } \\
\left(\mathrm{cm}^{2} / \mathrm{sec}\right)\end{array}$ & $\begin{array}{c}\text { CAV } \\
(\mathrm{cm} / \mathrm{sec})\end{array}$ & $\begin{array}{l}\text { VSI } \\
(\mathrm{cm})\end{array}$ & $\begin{array}{l}\mathrm{IH} \\
(\mathrm{m})\end{array}$ & $\begin{array}{c}\text { SMA } \\
\text { (g) }\end{array}$ & $\begin{array}{c}\text { SMV } \\
(\mathrm{cm} / \mathrm{sec})\end{array}$ & $\begin{array}{c}\text { EDA } \\
(\mathrm{g}) \\
\end{array}$ \\
\hline Cape Mendocino & 0.66 & 89.00 & 29.00 & 0.13 & 0.08 & 11.79 & 0.06 & 3.82 & 0.14 & 5049.2 & 1456.5 & 319.4 & 287.4 & 40.27 & 22.04 & 0.61 \\
\hline Coalinga & 0.73 & 37.50 & 5.20 & 0.05 & 0.05 & 2.87 & 70.01 & 1.44 & 0.07 & 238.5 & 447.7 & 7138.6 & 83.24 & 40.58 & 19.11 & 0.71 \\
\hline Coyote Lake & 0.43 & 49.20 & 7.70 & 0.11 & 0.04 & 5.15 & 0.01 & 0.77 & 0.05 & 761.9 & 433.1 & 150.2 & 136.50 & 0.16 & 13.75 & 0.41 \\
\hline Duzce & 0.72 & 56.44 & 23.10 & 0.07 & 0.07 & 8.33 & 0.06 & 3.72 & 0.12 & 3896.5 & 1478.6 & 234.8 & 211.20 & 0.44 & 40.53 & 0.61 \\
\hline Erzincan & 0.51 & 83.90 & 27.70 & 16 & 0.07 & 17.65 & 0.07 & 1.50 & 0.08 & 6665.6 & & 296.9 & 319.90 & 0.24 & 51.10 & 0.51 \\
\hline Friuli & 0.31 & 30.78 & 5.10 & 0.10 & 0.05 & 4.74 & 0.01 & 1.19 & 0.06 & 626.1 & 65 & 116.1 & 87.60 & 0.29 & 21.68 & 0.31 \\
\hline Gazli & 0.71 & 71.58 & 23.90 & 10 & 0.13 & 5.17 & 0.08 & 4.99 & 0.20 & 4142.4 & 1384.9 & 215.1 & 204.70 & 0.68 & 50.03 & 0.64 \\
\hline Irpinia & 0.37 & 114.5 & 89.60 & 0.30 & 0.06 & 24.27 & 0.24 & 2.87 & 0.10 & 35912.5 & 205 & 210.4 & 231.20 & 0.29 & 67.27 & 0.37 \\
\hline Kobe & 0.82 & 81.30 & 17.00 & 0.10 & 0.10 & 12.53 & 0.03 & 8.39 & 0.24 & 7589.6 & 2091.2 & 417.3 & 362.10 & 0.61 & 54.90 & 0.81 \\
\hline Landers & 0.78 & 31.88 & 16.00 & 04 & 0.09 & 5.01 & 0.03 & 6.58 & 0.19 & 1244.4 & 246 & 110.6 & 90.21 & 0.64 & 26.43 & 0.46 \\
\hline lomaperieta & 0.59 & 40.84 & 0.13 & 0.07 & 0.09 & 10.04 & 0.03 & 3.08 & 0.13 & 2589.2 & 125 & 219.5 & 190.50 & 0.39 & 37.70 & 0.50 \\
\hline imperial & 0.57 & 46.01 & 12.00 & 0.08 & 0.11 & 10.40 & 0.04 & 3.80 & 0.16 & 2434.0 & 1270.1 & 209.0 & 173.60 & 0.51 & 34.27 & 0.56 \\
\hline Morgan Hill & 0.31 & 39.60 & 6.40 & & 0.05 & 36 & 0.03 & 85 & 0.06 & & & 1.7 & 90 & 24 & 68 & 0.30 \\
\hline N. palm spring & 0.69 & 0.33 & 3.90 & 0.05 & 0.07 & 4.81 & 0.01 & 1.57 & 0.08 & 504.0 & 700.53 & 115.7 & 92.20 & 0.36 & 15.10 & 0.60 \\
\hline Nahanni & 0.95 & 42.60 & 9.30 & 0.05 & 0.12 & 8.17 & 0.03 & 4.41 & 0.18 & 1337.0 & 126 & 151.2 & 126.40 & 0.85 & 26.77 & 0.74 \\
\hline Jorthridge & 0.93 & 136.6 & 40.50 & & 0.17 & 25.92 & 0.09 & 7.38 & 0.28 & 10869.1 & 166 & 504.7 & 462.20 & 0.68 & 61.70 & 0.92 \\
\hline Parkfield & 0.47 & 75.00 & 22.40 & 0.16 & 0.05 & 7.64 & 0.03 & 1.78 & 0.07 & 3040.8 & 88 & 240.3 & 235.50 & 0.40 & 18.29 & 0.47 \\
\hline Tai & 0.24 & 26.10 & 11.40 & & 0.05 & 6.56 & 0.03 & 35 & 0.06 & 3.7 & 11 & 106.2 & 97.10 & 0.19 & 25.40 & 0.23 \\
\hline Victoria & 0.60 & 33.45 & 10.00 & 0.05 & 0.07 & 7.81 & 0.03 & 1.94 & 0.09 & 1492.5 & 982.2 & 159.3 & 141.20 & 0.41 & 28.17 & 0.46 \\
\hline Avaj & 0.47 & 93.20 & 23.00 & 0.20 & 0.05 & 8.32 & 0.02 & 7.03 & 0.14 & 14392.2 & 3402.9 & 396.6 & 380.70 & 0.43 & 74.28 & 0.44 \\
\hline Bam & 0.78 & 115.30 & 29.20 & & 0.09 & 10.74 & 0.03 & 7.90 & 0.21 & 7672.2 & 224 & 7397.0 & 389.00 & 0.63 & 40.83 & 0.68 \\
\hline Manjil & 0.45 & 32.58 & 19.50 & 0.07 & 0.07 & 6.94 & 0.10 & 4.17 & 0.13 & 2579.3 & 2424.90 & 161.4 & 144.70 & 0.42 & 26.33 & 0.41 \\
\hline Varzaqan & 0.47 & 105.60 & 40.50 & & 0.04 & 10.57 & 0.05 & 4.92 & 0.11 & 24839.8 & 2848 & 447.8 & 444.20 & 0.31 & 77.90 & 0.47 \\
\hline Chi-chi & 0.36 & 53.20 & 20.70 & 0.15 & 0.05 & 9.83 & 0.05 & 1.69 & 0.07 & 3832.4 & 917.' & 240.2 & 227.20 & 0.29 & 46.71 & 0.35 \\
\hline Tabas & 1.01 & 58.24 & 18.00 & 0.06 & 0.14 & 13.39 & 0.04 & 10.83 & 0.31 & 6642.0 & 2915 & 290.5 & 271.60 & 0.63 & 47.85 & 0.91 \\
\hline Firozabad & 1.07 & 143.13 & 49.00 & & 0.04 & 8.26 & 0.06 & 5.68 & 0.12 & 19418.8 & 2372 & 564.6 & 529.30 & 0.37 & 64.78 & 1.05 \\
\hline Karebas & 0.34 & 70.32 & 39.00 & 0.21 & 0.04 & 6.35 & 0.04 & 4.02 & 0.10 & 7722.3 & 2794.70 & 151.2 & 147.10 & 0.28 & 37.40 & 0.33 \\
\hline Sarein & 0.58 & 47.09 & 12.00 & 0.08 & 0.05 & 4.38 & 0.01 & 7.83 & 0.16 & 3939.9 & 3486.7 & 219.8 & 170.40 & 0.35 & 30.70 & 0.53 \\
\hline & 0.29 & 19.52 & 3.30 & & 0.03 & 2.58 & 0.01 & 0.52 & 0.03 & 266.0 & 540.1 & 67.05 & 53.39 & 0.20 & 10.47 & 0.29 \\
\hline Mammoth & 0.44 & 23.10 & 5.40 & 0.05 & 0.08 & 4.48 & 0.01 & 2.61 & 0.11 & 606.4 & $=1211.60$ & 94.13 & 85.13 & 0.35 & 14.50 & 0.34 \\
\hline
\end{tabular}

Rayleigh proportional damping [21] was considered as structural damping. The characterizations of seismic excitations used as inputs for the dynamic time history analyses are presented in Table 14.

It is necessary to mention that all of the selected seismic records are near-field earthquake records. Also, all of the seismic excitations have not been scaled. Seismic parameters related to each of the accelerogram are presented in Table 14. In next step, the two damage indices were calculated using output results obtained from the IDARC 7.0 software. Calculated values of the modified ParkAng damage indices and the maximum softening damage indices are presented in Table 15 and Table 16 respectively. Correlations between seismic parameters and damage indices were estimated by the Spearman correlation coefficient. For the modified Park-Ang model and the maximum softening model, values of the correlation coefficients are shown in Figures 2 to 17. 
Table 15. Values of the Overall Modified Park-Ang Model

\begin{tabular}{|c|c|c|c|c|c|c|}
\hline Earthquake & 5 story frame & 6 story frame & 7 story frame & 8 story frame & 9 story frame & 10 story frame \\
\hline Cape Mendocino & $1.01 \mathrm{E}-01$ & $1.33 \mathrm{E}+00$ & $3.30 \mathrm{E}-02$ & $4.50 \mathrm{E}-02$ & $1.88 \mathrm{E}-01$ & $6.00 \mathrm{E}-03$ \\
\hline Coalinga & 1.40E-02 & 1.30E-02 & 1.60E-02 & 1.20E-02 & 1.30E-02 & $1.71 \mathrm{E}+00$ \\
\hline Coyote Lake & 1.00E-03 & $1.00 \mathrm{E}-02$ & $1.37 \mathrm{E}+00$ & 6.00E-03 & $5.90 \mathrm{E}-01$ & 9.47E-01 \\
\hline Duzce & $1.05 \mathrm{E}+00$ & 4.90E-02 & 3.20E-02 & $1.48 \mathrm{E}+00$ & $1.20 \mathrm{E}-02$ & $1.22 \mathrm{E}+00$ \\
\hline Erzincan & 2.60E-02 & 1.00E-02 & 1.70E-02 & 1.40E-02 & $1.07 \mathrm{E}+00$ & 2.50E-02 \\
\hline Friuli & 5.00E-03 & 5.00E-03 & 5.90E-02 & 6.50E-01 & 2.40E-02 & 1.60E-02 \\
\hline Gazli & 4.00E-02 & $1.83 \mathrm{E}+00$ & $6.00 \mathrm{E}-03$ & 8.00E-03 & 1.07E-01 & 7.00E-03 \\
\hline Irpinia & 1.60E-02 & $1.19 \mathrm{E}+00$ & 1.70E-02 & 8.00E-03 & 1.40E-02 & 1.00E-02 \\
\hline Kobe & 1.23E-01 & 1.15E-01 & 1.47E-01 & 4.10E-02 & 3.12E-01 & 9.70E-02 \\
\hline Landers & $1.20 \mathrm{E}+00$ & $1.00 \mathrm{E}-02$ & $1.50 \mathrm{E}-02$ & 1.00E-03 & 1.30E-02 & $1.00 \mathrm{E}-03$ \\
\hline lomaperieta & 4.00E-02 & $1.89 \mathrm{E}+00$ & 1.70E-02 & 1.70E-02 & $1.50 \mathrm{E}-02$ & 1.80E-02 \\
\hline imperial & 1.61E-01 & 2.90E-01 & $1.61 \mathrm{E}+00$ & 5.00E-03 & $6.58 \mathrm{E}-01$ & 1.40E-02 \\
\hline Morgan Hill & 1.10E-02 & 1.10E-02 & 1.20E-02 & 1.60E-02 & 1.60E-02 & 1.90E-02 \\
\hline N. palm spring & 1.60E-02 & $1.70 \mathrm{E}-02$ & 1.10E-02 & 1.20E-02 & 8.50E-02 & $1.30 \mathrm{E}-02$ \\
\hline Nahanni & 1.40E-02 & $1.60 \mathrm{E}-02$ & 1.50E-02 & 1.50E-02 & $1.60 \mathrm{E}-02$ & $1.90 \mathrm{E}-02$ \\
\hline Northridge & 9.10E-02 & 1.13E-01 & 7.20E-02 & 8.00E-02 & $1.86 \mathrm{E}-01$ & 6.00E-02 \\
\hline Parkfield & 1.00E-02 & $3.50 \mathrm{E}-02$ & 4.40E-02 & 1.10E-02 & $1.13 \mathrm{E}+00$ & 1.50E-02 \\
\hline Taiwan & 3.30E-02 & 1.40E-02 & $1.60 \mathrm{E}-02$ & 1.40E-02 & 1.40E-02 & 1.00E-03 \\
\hline Victoria & $1.48 \mathrm{E}+00$ & $1.40 \mathrm{E}-02$ & $5.44 \mathrm{E}-01$ & $1.10 \mathrm{E}-02$ & $1.59 \mathrm{E}+00$ & $2.00 \mathrm{E}-02$ \\
\hline Avaj & 1.30E-02 & $1.94 \mathrm{E}+00$ & 7.00E-03 & $1.87 \mathrm{E}-01$ & 3.30E-02 & $1.39 \mathrm{E}-01$ \\
\hline Bam & 5.00E-03 & $9.50 \mathrm{E}-02$ & $1.27 \mathrm{E}+00$ & 8.57E-01 & $1.21 \mathrm{E}+00$ & 7.30E-02 \\
\hline Manjil & 4.30E-02 & $1.50 \mathrm{E}-02$ & $1.57 \mathrm{E}+00$ & 1.30E-02 & $5.74 \mathrm{E}-01$ & $1.80 \mathrm{E}-02$ \\
\hline Varzaqan & 2.80E-02 & 1.07E-01 & $2.90 \mathrm{E}-02$ & $5.80 \mathrm{E}-01$ & $1.15 \mathrm{E}-01$ & 5.80E-02 \\
\hline Chi-chi & 3.70E-02 & 1.30E-02 & $3.60 \mathrm{E}-02$ & $1.00 \mathrm{E}-02$ & $1.46 \mathrm{E}+00$ & $1.50 \mathrm{E}-02$ \\
\hline Tabas & 6.40E-02 & 8.30E-02 & $5.20 \mathrm{E}-02$ & 7.00E-03 & $1.20 \mathrm{E}+00$ & 5.80E-02 \\
\hline Firozabad & 2.50E-02 & 2.37E-01 & $1.40 \mathrm{E}-02$ & $1.60 \mathrm{E}-02$ & $2.06 \mathrm{E}-01$ & 1.40E-02 \\
\hline Karebas & $9.84 \mathrm{E}-01$ & $1.40 \mathrm{E}-02$ & $1.12 \mathrm{E}+00$ & $1.50 \mathrm{E}-02$ & 1.40E-02 & 1.70E-02 \\
\hline Sarein & 1.40E-02 & $1.54 \mathrm{E}+00$ & 3.60E-02 & $5.40 \mathrm{E}-02$ & $4.24 \mathrm{E}-01$ & $1.20 \mathrm{E}-02$ \\
\hline Chalfant & 1.10E-02 & 1.10E-02 & 1.40E-02 & 1.00E-03 & $0.00 \mathrm{E}+00$ & $0.00 \mathrm{E}+00$ \\
\hline Mammoth & $1.40 \mathrm{E}-02$ & $0.00 \mathrm{E}+00$ & $1.50 \mathrm{E}-02$ & 1.30E-02 & $1.00 \mathrm{E}-03$ & $0.00 \mathrm{E}+00$ \\
\hline
\end{tabular}

Table 16. Values of the Maximum Softening Damage Model

\begin{tabular}{|c|c|c|c|c|c|c|}
\hline Earthquake & 5 story frame & 6 story frame & 7 story frame & 8 story frame & 9 story frame & 10 story frame \\
\hline Cape Mendocino & $5.15 \mathrm{E}-01$ & $4.58 \mathrm{E}-01$ & $1.91 \mathrm{E}-01$ & $4.58 \mathrm{E}-01$ & 6.37E-01 & 2.31E-01 \\
\hline Coalinga & 2.76E-02 & 7.38E-02 & 4.44E-02 & 4.91E-01 & 3.00E-01 & 8.82E-01 \\
\hline Coyote Lake & 1.19E-03 & $1.53 \mathrm{E}-01$ & $8.18 \mathrm{E}-01$ & 1.43E-02 & 5.02E-01 & 8.32E-01 \\
\hline Duzce & $8.60 \mathrm{E}-01$ & 3.70E-01 & 5.30E-01 & 8.65E-01 & 2.72E-01 & 8.01E-01 \\
\hline Erzincan & $2.21 \mathrm{E}-01$ & 2.80E-01 & 3.68E-01 & $4.46 \mathrm{E}-01$ & 8.53E-01 & $2.48 \mathrm{E}-01$ \\
\hline Friuli & $1.28 \mathrm{E}-01$ & 1.20E-01 & 3.95E-01 & 6.33E-01 & 3.32E-01 & 2.17E-02 \\
\hline Gazli & 4.63E-01 & 3.41E-01 & 1.05E-01 & $1.27 \mathrm{E}-01$ & 6.75E-01 & $1.04 \mathrm{E}-01$ \\
\hline Irpinia & $1.07 \mathrm{E}-01$ & 8.49E-01 & 4.34E-01 & 6.37E-02 & $5.22 \mathrm{E}-01$ & $2.02 \mathrm{E}-01$ \\
\hline Kobe & 8.73E-01 & 7.81E-01 & 8.43E-01 & $3.44 \mathrm{E}-01$ & $5.40 \mathrm{E}-02$ & $3.34 \mathrm{E}-01$ \\
\hline Landers & $6.82 \mathrm{E}-01$ & $1.27 \mathrm{E}-01$ & $1.07 \mathrm{E}-01$ & $0.00 \mathrm{E}+00$ & $1.25 \mathrm{E}-01$ & $0.00 \mathrm{E}+00$ \\
\hline lomaperieta & 4.59E-01 & 3.69E-01 & $2.50 \mathrm{E}-01$ & 3.64E-02 & $0.00 \mathrm{E}+00$ & $1.51 \mathrm{E}-01$ \\
\hline imperial & $4.32 \mathrm{E}-01$ & 4.42E-01 & 8.57E-01 & 4.23E-02 & $5.22 \mathrm{E}-01$ & $1.02 \mathrm{E}-01$ \\
\hline Morgan Hill & $6.00 \mathrm{E}-02$ & 8.80E-02 & $2.71 \mathrm{E}-01$ & 3.72E-02 & $1.09 \mathrm{E}-01$ & 2.91E-01 \\
\hline N. palm spring & $1.78 \mathrm{E}-01$ & $1.65 \mathrm{E}-01$ & 2.55E-02 & 3.61E-02 & $1.87 \mathrm{E}-01$ & $1.69 \mathrm{E}-02$ \\
\hline Nahanni & 6.17E-03 & $1.04 \mathrm{E}-01$ & 2.70E-02 & 4.29E-02 & $5.46 \mathrm{E}-02$ & $2.32 \mathrm{E}-01$ \\
\hline Northridge & $5.75 \mathrm{E}-01$ & $4.68 \mathrm{E}-01$ & 7.91E-01 & 8.00E-01 & $6.44 \mathrm{E}-01$ & $5.48 \mathrm{E}-01$ \\
\hline Parkfield & $5.24 \mathrm{E}-01$ & 5.13E-01 & 5.15E-01 & 3.81E-02 & 8.35E-01 & 3.02E-01 \\
\hline Taiwan & $5.08 \mathrm{E}-01$ & 8.41E-02 & 2.07E-01 & 1.08E-03 & 5.42E-02 & $0.00 \mathrm{E}+00$ \\
\hline Victoria & 8.57E-01 & $2.00 \mathrm{E}-01$ & 6.35E-01 & 4.25E-02 & $9.16 \mathrm{E}-01$ & $1.55 \mathrm{E}-01$ \\
\hline Avaj & $2.24 \mathrm{E}-01$ & 8.16E-01 & $8.22 \mathrm{E}-02$ & $9.23 \mathrm{E}-01$ & 5.19E-01 & 6.82E-01 \\
\hline Bam & $1.53 \mathrm{E}-01$ & 2.51E-01 & 8.90E-01 & 8.14E-01 & 7.87E-01 & 2.31E-01 \\
\hline Manjil & 4.08E-01 & $3.26 \mathrm{E}-01$ & 9.01E-01 & 2.15E-03 & 5.67E-01 & 2.59E-01 \\
\hline Varzaqan & 4.49E-01 & 5.40E-01 & 4.73E-01 & 8.48E-01 & 9.16E-01 & 4.83E-01 \\
\hline Chi-chi & $4.55 \mathrm{E}-01$ & $1.53 \mathrm{E}-01$ & $5.42 \mathrm{E}-01$ & 1.03E-01 & 8.35E-01 & 2.91E-01 \\
\hline Tabas & $7.52 \mathrm{E}-01$ & 5.69E-01 & 5.96E-01 & 3.72E-02 & 8.76E-01 & $4.48 \mathrm{E}-01$ \\
\hline Firozabad & 3.53E-01 & $5.78 \mathrm{E}-01$ & $0.00 \mathrm{E}+00$ & 1.67E-01 & 8.67E-01 & 2.32E-01 \\
\hline Karebas & $9.22 \mathrm{E}-01$ & 2.03E-01 & 8.39E-01 & 4.65E-02 & 5.40E-02 & $2.78 \mathrm{E}-02$ \\
\hline Sarein & $1.27 \mathrm{E}-01$ & 8.22E-01 & 5.30E-01 & 3.69E-01 & 8.29E-01 & 2.46E-01 \\
\hline Chalfant & $1.28 \mathrm{E}-01$ & 1.07E-01 & 2.51E-01 & $0.00 \mathrm{E}+00$ & $0.00 \mathrm{E}+00$ & $0.00 \mathrm{E}+00$ \\
\hline Mammoth & $1.31 \mathrm{E}-01$ & $0.00 \mathrm{E}+00$ & $0.00 \mathrm{E}+00$ & $4.89 \mathrm{E}-02$ & $0.00 \mathrm{E}+00$ & $0.00 \mathrm{E}+00$ \\
\hline
\end{tabular}




\section{Discussion}

The determination of the interdependency between the overall structural damage index and seismic parameters can give a good indicator to predict the damaging potential of the earthquake record. The results of the Spearman correlation coefficients presented in Figures 2 to 17 have shown seismic parameters which have the strongest and the weakest correlation coefficients have been altered by the variation of number story. In fact, the strongest and the weakest correlation coefficients change with the variation of structural stiffness. For five, six, seven, eight, nine, and ten story concrete frames, the modified Park-Ang model had the strongest correlations with the characteristic intensity, VSI, $\mathrm{V}_{\max } / \mathrm{A}_{\max }$, VSI, VSI, and EDA respectively.

On the other hand, for the overall modified Park-Ang model, $\mathrm{V}_{\max } / \mathrm{A}_{\max }$, ARMS, PGA, VRMS, SMA, and CAV showed the fairest interdependencies with five, six, seven, eight, nine, and ten story frames respectively. As it is presented in Figures 2 to 17, among all of the reinforced concrete frames, six story frame has the highest range of the correlation values between all of the seismic parameters and the overall modified Park-Ang model.

As it is presented in Figures 2 to 17, for five, six, seven, eight, nine, and ten story concrete frames, the maximum softening damage index has the strongest correlations with characteristic intensity, the Housner intensity, $\mathrm{V}_{\max } / \mathrm{A}_{\max }$, VSI, VSI, and EDA respectively. On the contrary, for the maximum softening damage index, $\mathrm{V}_{\max } / \mathrm{A}_{\max }$, ARMS, PGA, ARMS, ARMS, and CAV presented the fairest correlation with five, six, seven, eight, nine, and ten story frames respectively.

Also, among all of the reinforced concrete frames, the correlation values of seismic parameters presented in Figures 2 to 17 have shown that the highest range of correlation values was related to six story concrete frames.

Among all of the seismic parameters, Velocity Spectrum Intensity (VSI) has strong interdependencies with the overall modified damage index and the maximum softening damage index for all of the structural concrete frames.

Consequently, the value of Velocity Spectrum Intensity (VSI) can be used as a good indicator to predict damaging potential of the earthquake record. Velocity Spectrum Intensity (VSI) presents the intensity of shaking of an earthquake at a region.

Velocity Spectrum Intensity (VSI) is defined as the area under elastic velocity spectrum between the periods 0.1 and $2.5 \mathrm{~s}$. In fact, values of correlations showed that spectral and energy parameters have strong correlation with the maximum softening damage index and the overall modified Park-Ang model.

As shown in Figures 2 to 17, regular variations are not attained by variations of the story numbers for each of the seismic parameters. Also, for five story frame, all of the correlation values between the ParkAng model and seismic parameters have shown the stronger correlations than all of the correlation values between the maximum softening model and seismic parameters. On the other hand, for eight and ten story frame, all of the correlation values between the maximum softening model and seismic parameters have presented the stronger correlations than correlation values between the Park-Ang model and seismic parameters.

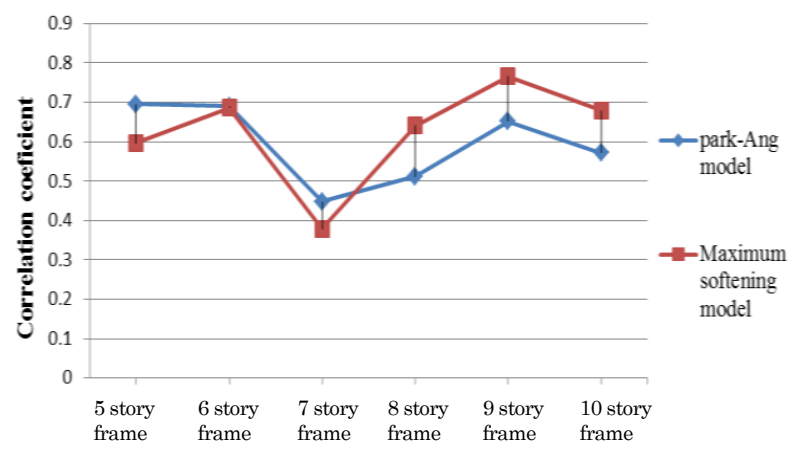

Figure 2. Comparative Diagram for PGA Parameter

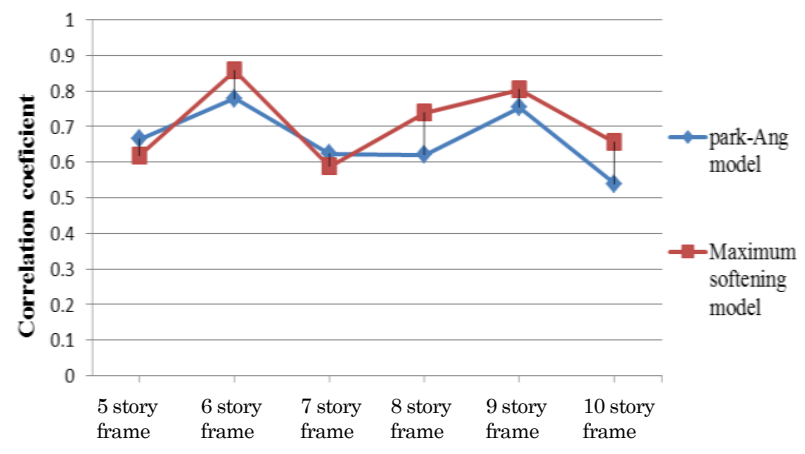

Figure 3. Comparative Diagram for PGV Parameter

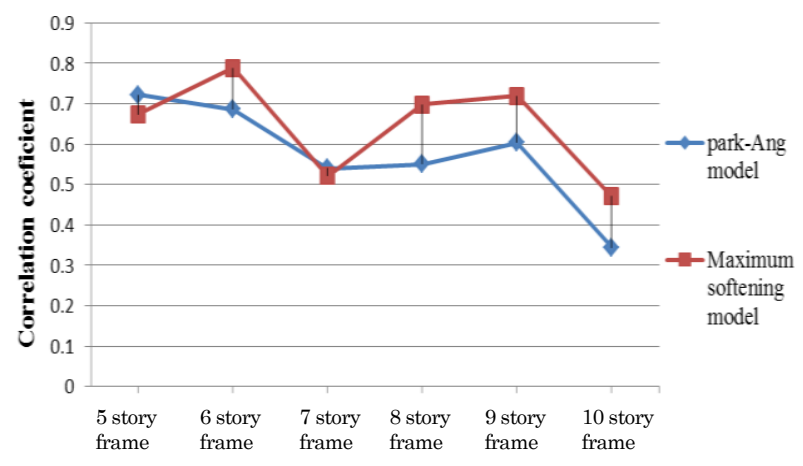

Figure 4. Comparative Diagram for PGD Parameter 


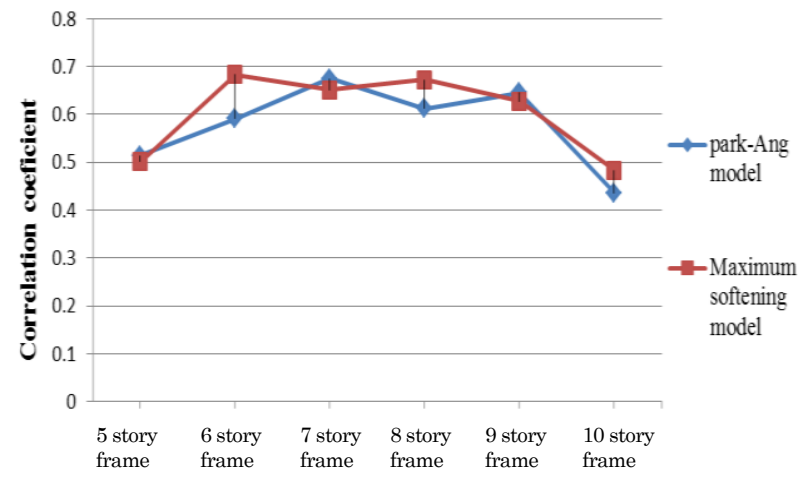

Figure 5. Comparative Diagram for $\mathrm{V}_{\max } / \mathrm{A}_{\max }$ Parameter

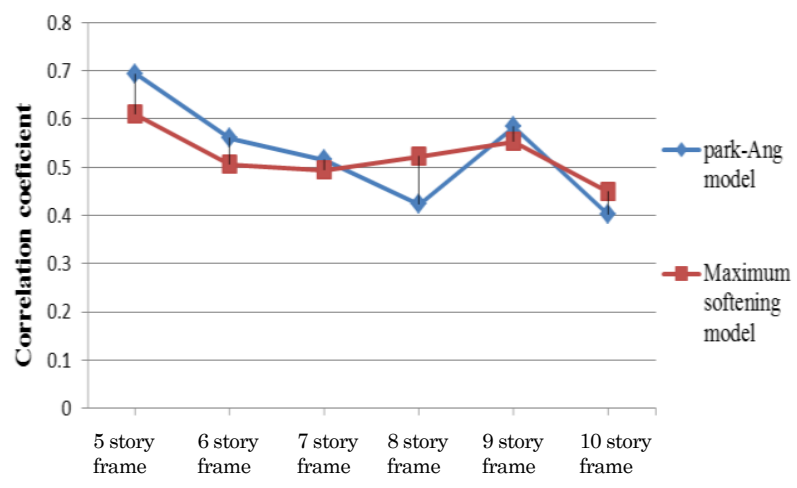

Figure 6. Comparative Diagram for Acceleration RMS Parameter

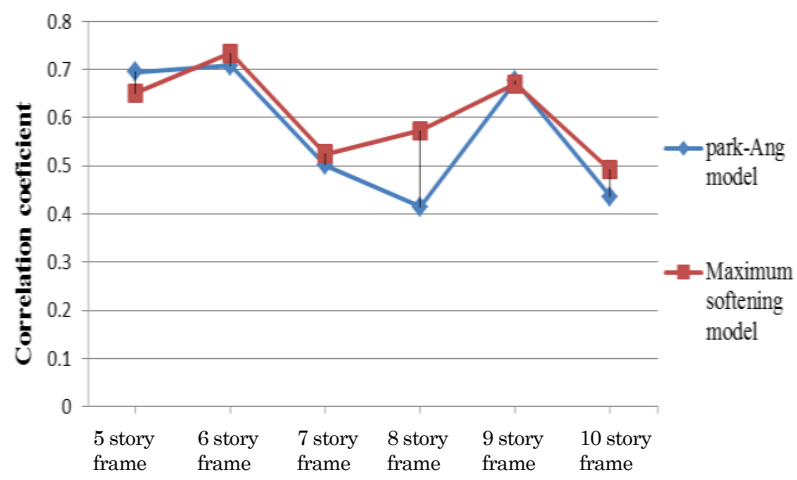

Figure 7. Comparative Diagram for Velocity RMS Parameter

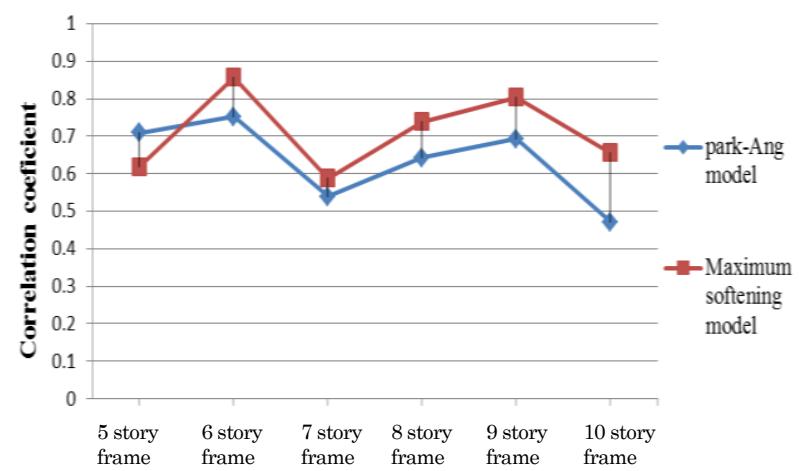

Figure 8. Comparative Diagram for Displacement RMS Parameter

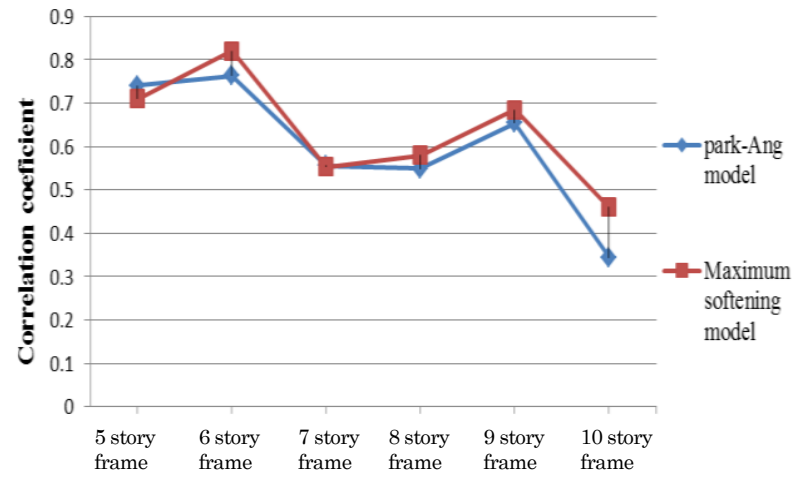

Figure 9. Comparative Diagram for Arias Intensity Parameter

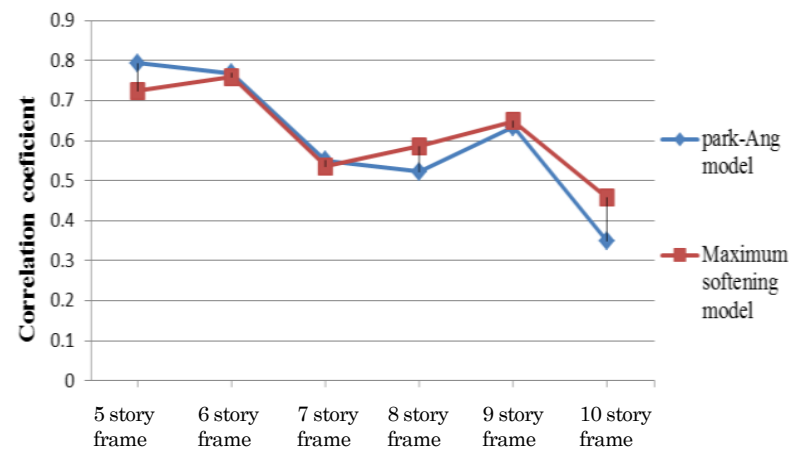

Figure 10. Comparative Diagram for Characteristic Intensity Parameter

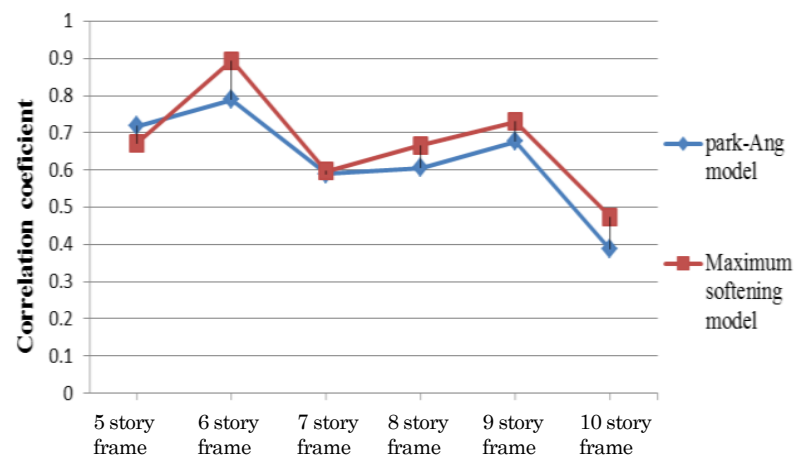

Figure 11. Comparative Diagram for Specific Energy Density Parameter

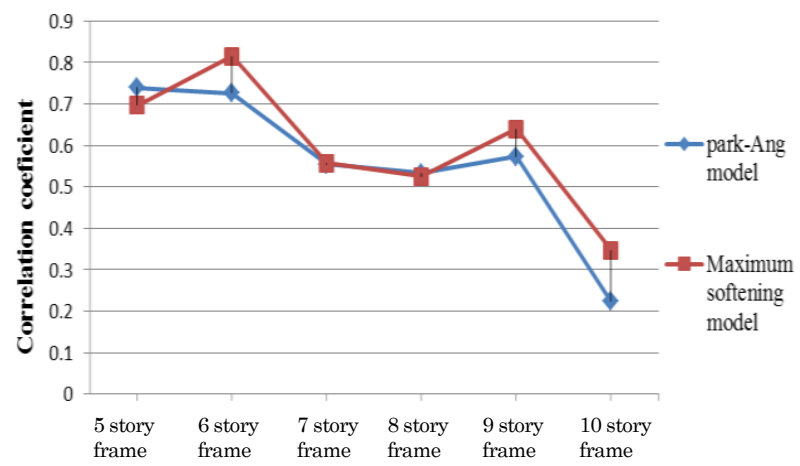

Figure 12. Comparative Diagram for Cumulative Absolute Velocity Parameter 


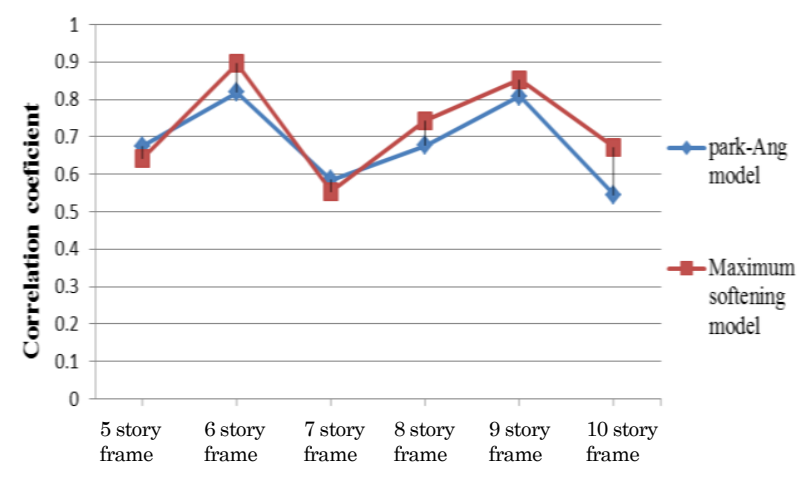

Figure 13. Comparative Diagram for Velocity Spectrum Intensity Parameter

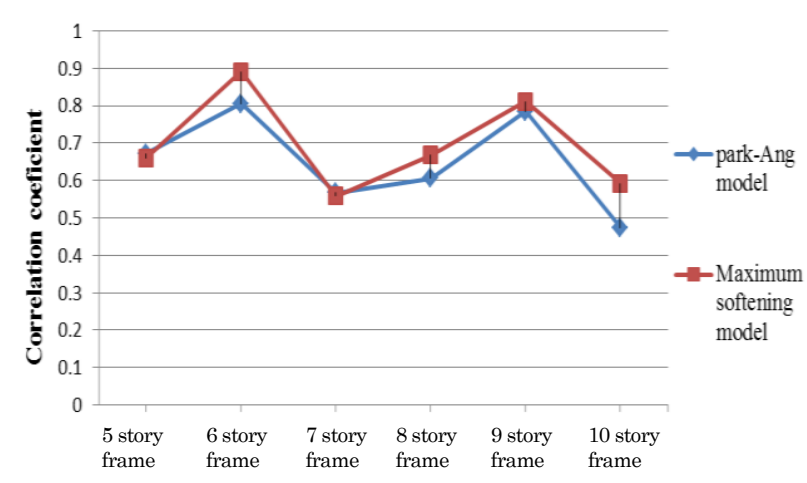

Figure 14. Comparative Diagram for Housner Intensity Parameter

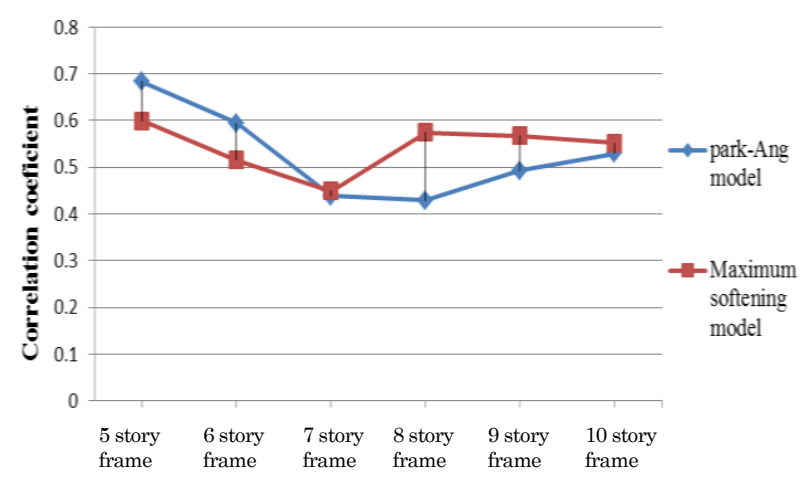

Figure 15. Comparative Diagram for Sustained Maximum Acceleration Parameter

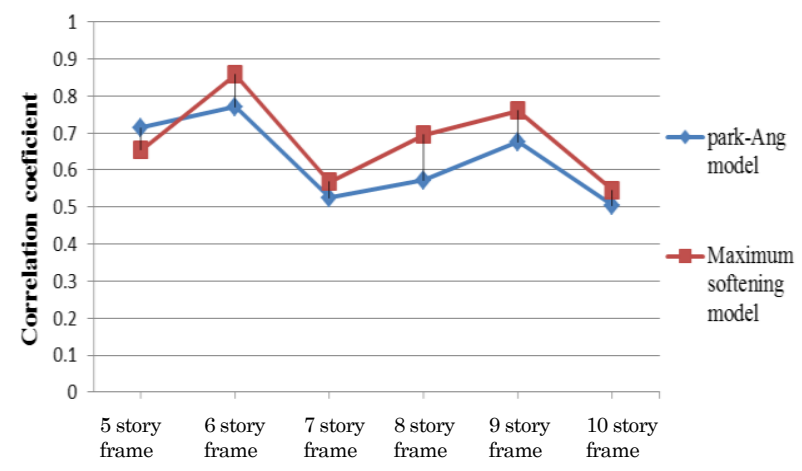

Figure 16. Comparative Diagram for Sustained Maximum Velocity Parameter

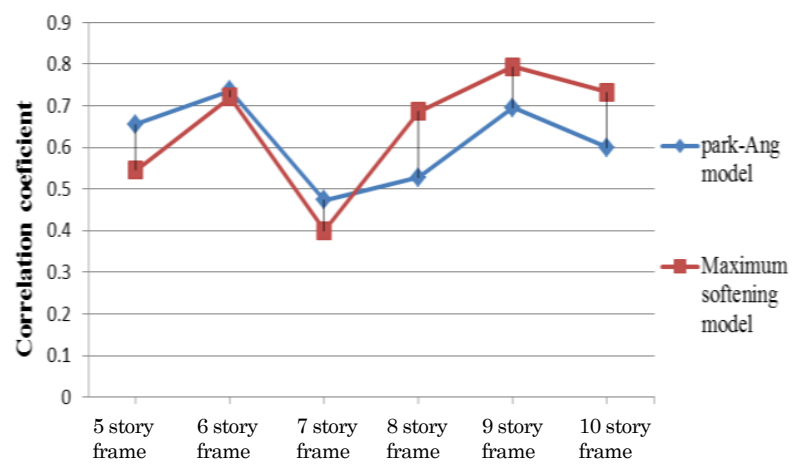

Figure 17. Comparative Diagram for Effective Design Acceleration Parameter

\section{Conclusion}

In this paper, determination of interdependencies between seismic parameters and two overall damage indices; the modified Park-Ang model and the maximum softening damage index were investigated by the Spearman correlation coefficient. For this purpose, 30 near-field earthquake records were selected from all over the world. Also, six different types of reinforced concrete frames were utilized for nonlinear dynamic analyses.

Among all of the seismic parameters, the results have shown that spectral and energy parameters have strong correlation with the maximum softening damage index and the overall modified Park-Ang model.

Generally, Velocity Spectrum Intensity has presented a strong correlation with two overall structural damage indices (the maximum softening damage and the modified Park-Ang model) for all of the reinforced concrete frames. Therefore, as the numerical results of correlations have shown, Velocity Spectrum Intensity (VSI) can be used as a good indicator to predict the damaging potential of earthquake record. In the end, among all of the structural concrete frames, six story frames presented the highest range of correlation values between all of the seismic parameters and two overall damage indices; the maximum softening damage and the modified Park-Ang model.

\section{Acknowledgement}

The supports by Shahid Abbaspour Campus of Shahid Beheshti University are gratefully appreciated.

\section{References}

1. DiPasquale, E. and Cakmak, A.S., On the Relation between Local and Global Damage Indices, Technical Report NCEER-89-0034, State University of New York at Buffalo, 1989. 
2. Park, Y.J., Ang, A.H.S., and Wen, Y.K., Damage Limiting a seismic Design of Building, Journal of Earthquake Spectra, 3(1), 1987, pp. 1-25.

3. Kennedy, R.P., Ground Motion Parameter useful in Structural Design, Proceeding of the Conference on Evaluation of Regional Seismic Hazards and Risk, New Mexico, 1980, pp. 83-95.

4. Nuttli, O.W., The Relation of Sustained Maximum Ground Acceleration and Velocity to Earthquake Intensity and Magnitude, US Army Corps of Engineers, Waterways Experiment Station, Mississippi; Report 16, 1979, pp. 71-81.

5. Newmark, N.M. and Hall, W.J., Earthquake Spectra and Design, EERI Monograph. Earthquake Engineering Research Institute Berkeley, California, 1982, pp. 103-104.

6. Benjamin, J.R., A Criterion for Determining Exceedance of the Operating Basis Earthquake, Electric Power Research Institute, Palo Alto, California, EPRI Report NP-5930, 1998.

7. Arias, A., A Measure of Earthquake Intensity in Seismic Design for Nuclear Power Plants, MIT Press, Cambridge, 1970, pp. 438-483.

8. Maniyar, M.M. and Khare, R.K., Selection of Ground Motion for Performing Incremental Dynamic Analysis of Existing Reinforced Concrete Buildings in India, Journal of Current Science, 100, 2011, pp. 5-18.

9. Elenas, A., Correlation Between Seismic Acceleration Parameters and Overall Structural Damage Indices of Buildings, Journal of Soil Dynamics and Earthquake Engineering, 20, 2000, pp. 93-100.

10. Trifunac, M.D and Brady, A.G., A Study on the Duration of Strong Earthquake Ground Motion, Bulletin of the Seismological Society of America, 65(3), 1975, pp. 581-626.

11. Elenas, A. and Meskouris, K., Correlation Study between Seismic Acceleration Parameters and Damage Indices of Structures, Journal of Engineering Structures, 23, 2001, pp. 698-704.

12. Nanos, N., Elenas, A., and Ponterosso, P., Correlation of Different Strong Motion Duration
Parameters and Damage Indicators of Reinforced Concrete Structures, Proceeding of the Conference on Earthquake Engineering, China, Beijing, October 12-17, 2008.

13. Elenas, A., Intensity Parameters as Damage Potential Descriptors of Earthquakes, Proceeding of the Conference on Computational Methods in Structural Dynamics and Earthquake Engineering, Greece, Corfu, 2011.

14. Safi, M. and Soleymani, A., Investigation of Correlations between Seismic Parameters and Damage Indices for Earthquake of Iran Region, International Journal of Engineering, 27(2), 2014, pp. 291-300.

15. Estekanchi, H. and Arjomandi, K., Correlation of Damage Indices in Nonlinear Time History Analysis of Steel Moment Frames, Asian Journal of Civil Engineering (Building and Housing), 8, 2007, pp. 629-646.

16. Park, Y.J. and Ang, A.H.S., Mechanistic Seismic Damage Model for Reinforced Concrete, American Society of Civil Engineering, Journal of Structural Engineering, 111(4), 1985, pp. 722-739.

17. Spiegel, M.R., Theory and Problems of Statistics, McGraw-Hill, England, London, 1992.

18. Building Code Requirements for Structural Concrete (ACI 318-02) and Commentary (ACI318R-02), American Concrete Institute, Farmington Hills, Michigan.

19. Uniform Building Code (UBC), International Conference of Building Officials, Whittier, California, 1997.

20. Kunnath, S.K., Reinhorn, A.M., and Lobo, R.F., IDARC Version 3.0: A Program for the Inelastic Damage Analysis of Reinforced Concrete Structures, Report No. NCEER-92-0022, National Center for Earthquake Engineering Research, University at Buffalo, The State University of New York, 1992.

21. Liu, M. and Gorman, D.G., Formulation of Rayleigh Damping and its Extension, Journal of Computers \& Structures, 57(2), 1995, pp. 277 285. 